\title{
Chemical synthesis of graphene nanoribbons
}

\author{
Eleftherios K. Pefkianakis, ${ }^{\text {a,b }}$ Georgios Sakellariou, ${ }^{\text {b }}$ and Georgios C. Vougioukalakis ${ }^{* a}$ \\ ${ }^{a}$ Laboratory of Organic Chemistry, Department of Chemistry, University of Athens, \\ Panepistimiopolis, GR-15771 Athens, Greece \\ ${ }^{b}$ Laboratory of Industrial Chemistry, Department of Chemistry, University of Athens, \\ Panepistimiopolis, GR-15771 Athens, Greece \\ E-mail:vougiouk@chem.uoa.gr
}

\section{Dedicated to Professor Michael Orfanopoulos on the occasion of his $67^{\text {th }}$ birthday and his official retirement}

DOI: http://dx.doi.org/10.3998/ark.5550190.p008.995

\begin{abstract}
Graphene is a two-dimensional atom-thick sheet of graphite composed of an $s p^{2}$-hybridized carbon atom network. Its isolation in 2004 and the extensive research that followed have led, amongst others, to graphene nanoribbons (GNRs), a graphene-based structure having nano-scale dimensions and semiconducting or metallic electronic properties that depend on its geometry and dimensions. These characteristics of GNRs are in stark contrast to those of graphene, which is a carbon sheet with semimetal, zero band gap characteristics. In the present article, we discuss the progress that has been reported towards producing GNRs with predefined dimensions, by using bottom-up chemical synthesis approaches.
\end{abstract}

Keywords: Graphene, nanoribbons, bottom-up synthesis, nano-structures

\section{Table of Contents}

1. Introduction

2. GNR Synthesis via bottom-up approaches

2.1 GNR synthesis in solution via coupling reactions of organic building-blocks

2.2 GNR synthesis via the conversion of precursors inside CNTs

2.3 GNR synthesis through surface-assisted polymerization

3. Conclusions

4. Acknowledgements

References 


\section{Introduction}

Carbon-based materials have attracted the interest of both academia and industry owing to their extraordinary physical and chemical properties that make them suitable for many cutting-edge technological applications. The most well-studied carbon-based materials are fullerenes $\left(\mathrm{C}_{60}\right.$ and its higher derivatives), ${ }^{1-3}$ carbon nanotubes (CNTs) ${ }^{4-6}$ graphene $^{7,8}$ and graphene derivatives such as graphene oxide $(\mathrm{GO})^{9}$ and graphene nanoribbons (GNRs), ${ }^{10}$ carbon nanodots (CDs), ${ }^{11}$ and carbon fibers. ${ }^{12}$ To date, the only successfully commercialized material are carbon fibers, which are industrially produced on a large scale for applications where high tensile strength and rigidity are needed (e.g., in polymer reinforcement). The rest of the aforementioned carbon materials have, nevertheless, found many optoelectronic and analytical applications, but their efficiency is either limited or their production costs are high, making their large-scale applications inexpedient. In terms of scientific interest, fullerenes and their derivatives have, amongst others, been successfully incorporated in organic photovoltaics, as the electron-accepting material. ${ }^{13,14}$ Carbon nanotubes have also found application in photovoltaics, as the electron-accepting moiety or as the core material of the electrodes, as well as in the reinforcement of a variety of materials. ${ }^{6,15,16}$ In the last decade, graphene and its derivatives have attracted the interest of the scientific community and are considered as promising candidates for the replacement of silicon in future electronics. ${ }^{17-19}$

Graphene is a two-dimensional, atom-thick sheet of graphite composed of a continuous hexagonal network of $s p^{2}$-hybridized carbon atoms, yet without well-defined edges and with many discontinuities. Since its isolation in 2004 by Novoselov et al., ${ }^{20}$ who reported a micromechanical cleavage approach for the exfoliation of graphite based on repeated peeling of highly-oriented pyrolyzed graphite (HOPG) with scotch tape, many research groups entered the fields of graphene and graphene oxides chemistry and physics. Pioneering studies were conducted by Novoselov and Geim, leading to the recognition of their work with the award of the 2010 Nobel Prize in Physics. ${ }^{7,8}$ Nevertheless, the absence of a procedure for the controllable and reproducible synthesis of graphene sheets with well-defined size, shape, and edge structure, characteristics that would predefine their electronic properties, remains a critical issue limiting their applicability in optoelectronics. GNRs are materials that could resolve these problems as they are theoretically predicted to have tailor-made physical properties. ${ }^{21-23}$

More specifically, GNRs are predicted to exhibit electronic properties that are dictated by the ribbon width and the shape of the edges. ${ }^{24,25}$ In terms of their edge structure, GNRs can be categorized as zigzag- or armchair-edged (Scheme 1). The difference between these two GNR types is that zigzag-edged GNRs possess a localized nonbonding $\pi$ state around the zigzag edges, while armchair-edged GNRs lack such a state. The localized nonbonding $\pi$ state is predicted to yield specific magnetic activities in GNRs. This peculiar electron localization, along with the symmetry-broken spatial spin distribution in zigzag GNRs, is referred to as the "edge state". Zigzag GNRs are predicted to be always metallic, whereas armchair GNRs can be metallic or semiconducting depending on their width. ${ }^{24,25}$ 


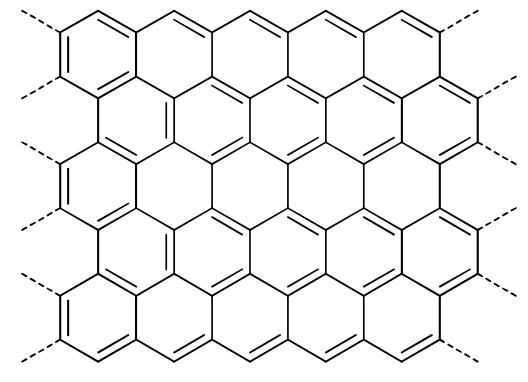

Zigzag

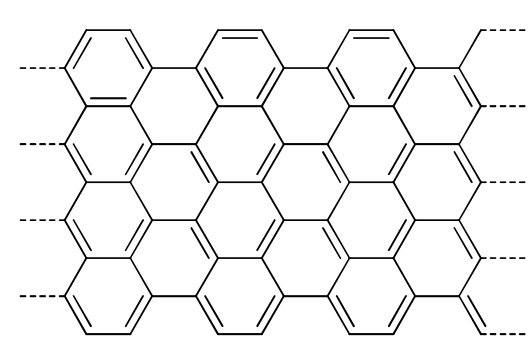

Armchair

Scheme 1. Schematic representation: zigzag-edged and armchair-edged GNRs.

To date, GNRs are obtained by following two very different strategies, namely the top-down (the breaking down of large preformed carbon-based structures) and the bottom-up (the assembly of simple building blocks into a complex structure). Top-down techniques involve the unzipping of carbon nanotubes using an e-beam ${ }^{26}$ or via chemical oxidation using potassium permanganate and sulfuric acid, ${ }^{27}$ by intercalating lithium into MWCNTs followed by thermal expansion, ${ }^{28}$ and by longitudinal splitting of MWCNTs using transition metal clusters. ${ }^{29}$ GNRs have also been prepared via the etching of graphene by lithography, ${ }^{30}$ by plasma chemical vapor deposition (CVD) under a $\mathrm{Ni}$ bar, ${ }^{31}$ via mechanical exfoliation of $\mathrm{HOPG}^{20}$ through solution-based exfoliation of graphite intercalation compounds (GICs), ${ }^{18}$ by chemical oxidation/exfoliation of graphite followed by reduction of the resulting graphene oxide (GO), ${ }^{32-35}$ as well as resist-based patterning ${ }^{17}$ and scanning tunneling microscopy (STM) patterning. ${ }^{36}$ While top-down methods have the advantage of producing GNRs with micrometer length, they frequently involve complicated procedures, they lack reproducibility, and the final materials have poorly-defined chiralities. Furthermore, top-down-produced GNRs' edge structures feature a large number of defects and the width of the ribbon can be larger than the molecular dimensions.

On the other hand, bottom-up techniques can afford GNRs with distinct structural and geometrical characteristics (defined edge type and narrow widths), without edge abnormalities, very low polydispersity, and the potential for scale-up. ${ }^{10,37-39}$ Importantly, the precursor monomers used to chemically synthesize the GNRs define the ribbon dimensions, which in turn dictates the band gaps and electronic properties of the final material. On this basis, producing GNRs with tailor-made predefined widths, edge structure geometries and suitable solubility constitutes an important challenge for synthetic chemists.

Bottom-up approaches reported to date include organic synthesis in solution via crosscoupling of the appropriate organic building-blocks followed by the dehydrogenation of the resulting oligomers, ${ }^{37,38,40-47}$ the conversion of precursors inside CNTs, ${ }^{48,49}$ and surface-assisted polymerization with subsequent dehydrogenation in an ultra-high vacuum environment. ${ }^{39,50,51}$ Although these bottom-up methods provide GNRs with a defined edge structure, they so far suffer from the inability to afford GNRs having variable widths at large scales, owing to the low solubility of the synthesized GNRs, and/or the need for highly-specialized instrumentation. 


\section{GNR Synthesis via bottom-up approaches}

Attempts to chemically obtain graphene nanoribbon-like materials by synthetic chemistry had been reported before the isolation and characterization of parent graphene. Müllen and coworkers ${ }^{52}$ pioneered this area, having reported the synthesis of a number of polycyclic aromatic hydrocarbons (PAHs). The production of these early PAHs was based either on Diels-Alder or cyclotrimerization reactions of suitable precursor molecules. Then, the final materials were obtained by oxidative cyclodehydrogenation of the respective oligophenylene precursors under mild conditions. The as-obtained 2-D graphitic materials had electronic properties similar to those of macroscopic graphite. At the time, owing to the poor solubility of these materials in common organic solvents, the complete structural characterization of these materials was not possible: characterization was performed via laser desorption/ionization time-of-flight mass spectrometry and UV/visible spectroscopy of the corresponding PAHs thin films. Nevertheless, this work paved the way, so that eight years later the same research group published results that led to the establishment of the bottom-up approach for the synthesis of GNRs. ${ }^{40}$

\subsection{GNRs synthesis in solution via coupling reactions of organic building-blocks}

In 2008, Müllen and co-workers ${ }^{40}$ reported a new method for the synthesis of linear, 2-D graphene nanoribbons with lengths of up to $12 \mathrm{~nm}$ (Scheme 2, GNR1). These nanoribbons were produced in good yields, via the Suzuki-Miyaura coupling polymerization between a suitable diiodo-modified tetraphenylbenzene (compound 1, Scheme 2) and a diboronic ester modified hexaphenylbenzene (compound 2, Scheme 2), followed by a Scholl (dehydrogenation) reaction. The as-obtained GNRs had lengths ranging from 8 to $12 \mathrm{~nm}$ and were carrying dimethyl-octyl substituents on their edges to improve solubility and, therefore, overcome problems observed with the previously-synthesized PAHs. ${ }^{52}$ This attempt was quite successful and characterization of the resulting GNRs was carried out by mass spectrometry, UV/Vis spectroscopy, scanning electron microscopy (SEM), transmission electron microscopy (TEM), and scanning tunneling microscopy (STM). Moreover, these 2-D carbon structures exhibited a high tendency to selfassemble in a regular lamellar pattern. 

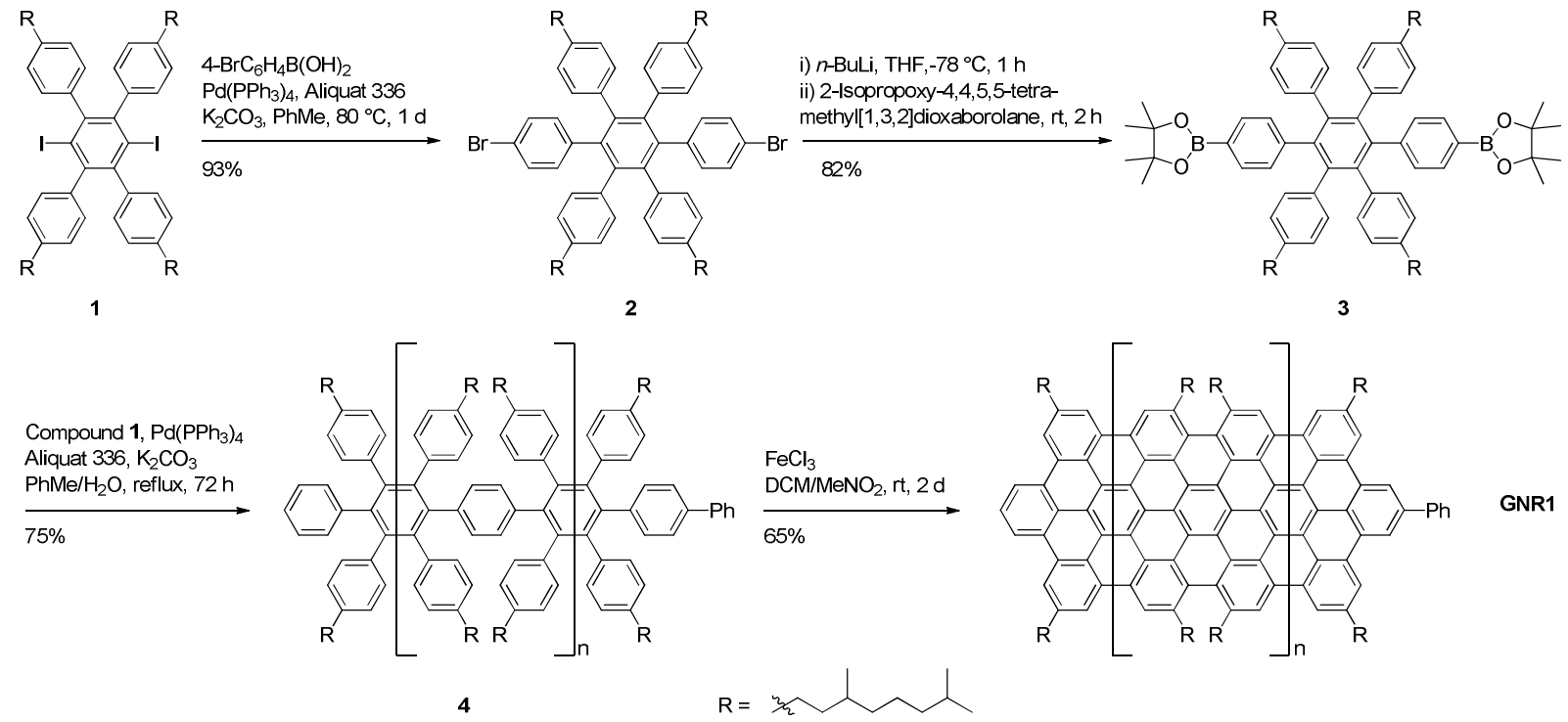

Scheme 2. Müllen's synthetic route for the first chemically synthesized GNR1. ${ }^{40}$

In the same year, Wang, Negri and co-workers ${ }^{41}$ reported the use of perylene bisimides (PBIs) for the production of functionalized graphene nanoribbons. Their decision to utilize PBIs was based on: i) the fact that this class of organic molecules shows versatile optical and electrochemical properties; ii) the possibility to fine-tune these properties by attaching various substituents at their bay positions; iii) their high electron affinity; and iv) their chemical and thermal stability. All these characteristics make PBIs attractive n-type materials in electronics and optoelectronics. The authors synthesized and characterized fully conjugated tri(perylene bisimides), having 19 six-membered carbon rings in the core and imide groups at the edges (Scheme 3), by utilizing a copper-mediated coupling reaction of a tetrabromo-substituted PBI precursor (compound 5, Scheme 3). This coupling reaction produced two isomeric structures of triPBIs (Scheme 3, GNRs 2 and 3), which were successfully separated via HPLC. Their structural characterization was performed by means of ${ }^{1} \mathrm{H}$ NMR and MALDI-TOF mass spectrometry. Having 19 benzene rings and 6 imide groups in their structure, the obtained triPBIs display broad and red-shifted absorption spectra along with six reduction waves in their cyclic voltammograms. By comparing these triPBIs with PBIs and diPBIs, the authors concluded that by increasing the number of PBI units in oligo-PBIs (from PBIs to diPBIs and eventually triPBIs) the resulting expansion of the $\pi$ system leads to: i) the reduction of the transport and optical band gaps; ii) the lowering of LUMO energies; iii) a mild reduction of the ionization potentials; and iv) a remarkable increase in electron affinities, all suggesting the potential applications of triPBIs in organic electronics. 


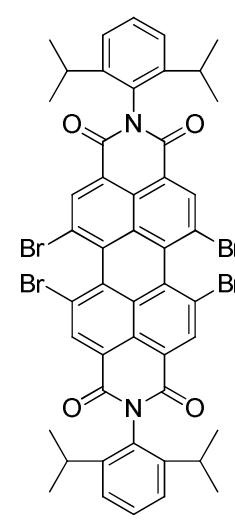

Cul, L-proline, $\mathrm{K}_{2} \mathrm{CO}_{3}$ DMSO, $12 \mathrm{~h}$

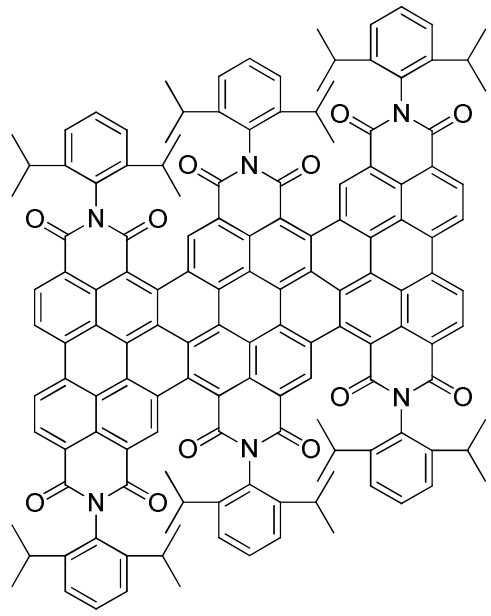

GNR2

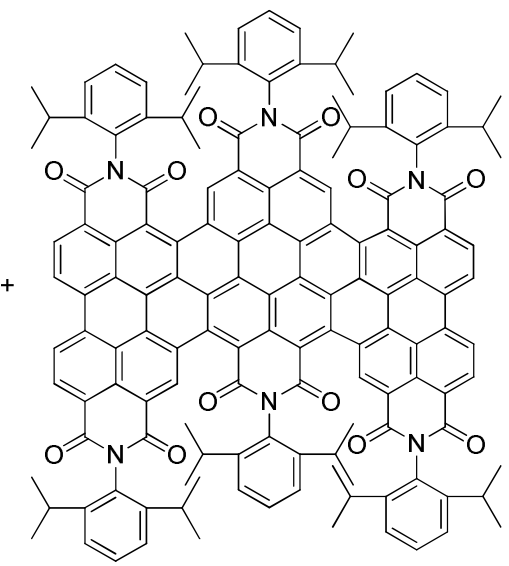

GNR3

Scheme 3. Bottom-up synthesis of functionalized GNRs 2 and 3 by Wang, Negri and coworkers. $^{41}$

Related work by Müllen and collaborators ${ }^{42}$ used microwave assisted Diels-Alder reactions to obtain five monodisperse ribbon-type polyphenylenes with rigid dibenzo[e,l]pyrene cores in their repeat units. These polyphenylenes served as precursors for giant PAH ribbons and were variable in terms of the carbon atoms present in the aromatic backbone, ranging from 132 to 372 carbon atoms and, moreover, incorporated up to six dibenzo[ $e, l]$ pyrene units, thus showing quite different aspect ratios (Scheme 4, GNR4). Additionally, dodecyl chains attached at the periphery of the backbone, aided solubility in common organic solvents and enabled their full characterization by standard analytical techniques. All synthesized PAH ribbon precursors, having 132, 192, 252, 312 and 372 carbon atoms in their backbone, respectively, were characterized by MALDI-TOF mass spectrometry and NMR spectroscopy. Gel permeation chromatography showed that these polyaromatic compounds were monodisperse, which is of outmost importance for the final application of such materials, while high-performance liquid chromatography supported the purity of the products. The next step in producing the nanoribbons was a cyclodehydrogenation (Scheme 4). For this step, the incorporated dibenzo[e,l]pyrene cores were an important feature, since they facilitated the dehydrogenation which improved the reaction yields. When the GNRs were eventually produced, their structure could not be verified owing to poor solubility, despite the attachment of the dodecyl moieties at their edges; MALDITOF mass spectrometry was successful only for the smallest homologue, and for the higher molecular weight ribbons extensive fragmentation occurred during the measurements. STM studies revealed that when adsorbed on highly oriented pyrolytic graphite (HOPG) these graphitic molecules self-organize into 2-D columns. As a result, they are attractive candidates for applications in organic electronics such as field effect transistors. From a synthesis perspective, an interesting feature of Müllen's method was that the stoichiometrically controlled Diels-Alder 
reaction gave two important compounds in a single step: the homologue of the desired series (compounds 12 or 14, Scheme 5) and the starting compound for the next higher homologue (compounds 11 or 13, Scheme 5), as shown in Scheme 5 for the case of the first two and smallest precursor molecules as an example.

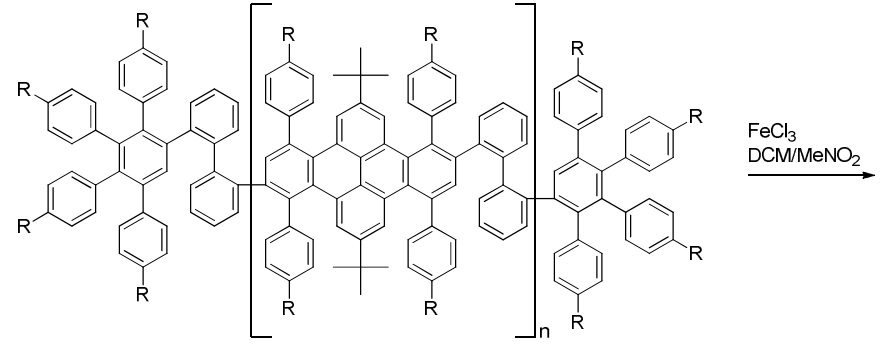

$n=1,2,3,4,5$ and equals to $132,192,252,312$ and 372 carbon atoms in the backbone

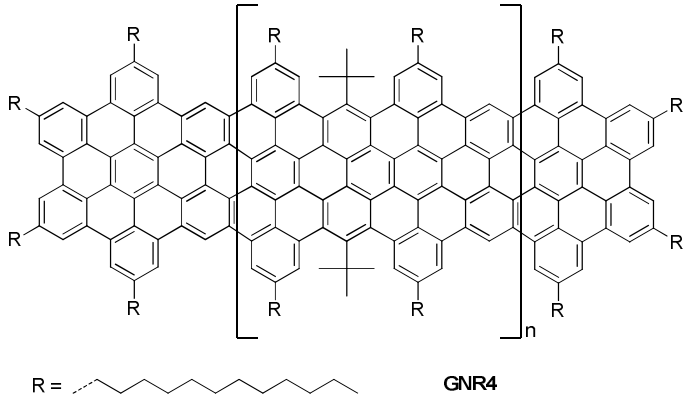

GNR4

Scheme 4. Cyclodehydrogenation of the precursor molecules to afford the final GNR4 type materials. $^{42}$

Two years later, the same research group reported the synthesis of a series of nanoribbons derived from polyphenylene precursors with a non rigid kinked backbone (Scheme 6, GNR5a-c type $)^{43}$ that showed higher solubility compared to the previously reported rigid linear poly(paraphenylene) systems. ${ }^{42,52}$ For the preparation of the precursor polymers, microwave assisted Suzuki-Miyaura coupling polymerization was used. The absence of backbone rigidity facilitated the synthesis of high molecular weight polymers (up to $16.000 \mathrm{~g} \mathrm{~mol}^{-1}$ ). The kinked backbone polymers obtained were converted into the corresponding nanoribbons via an intramolecular cyclodehydrogenation reaction, using $\mathrm{FeCl}_{3}$ as the oxidant in dichloromethane at room temperature for 3 days. The final GNRs, with lengths up to $40 \mathrm{~nm}$ (Scheme 6), hosted dodecyl substituents at their periphery and were obtained as waxy materials that dissolved in common organic solvents, such as toluene, tetrahydrofuran, and dichloromethane. One GNR analogue that was synthesized without the dodecyl chain decoration was obtained as an insoluble powder. The enhanced solubility of the dodecyl-substituted GNRs enabled their detailed structural characterization by means of Raman spectroscopy, MALDI-TOF mass spectrometry, and solution techniques, such as UV/Vis absorption, photoluminescence, and gel permeation chromatography. 

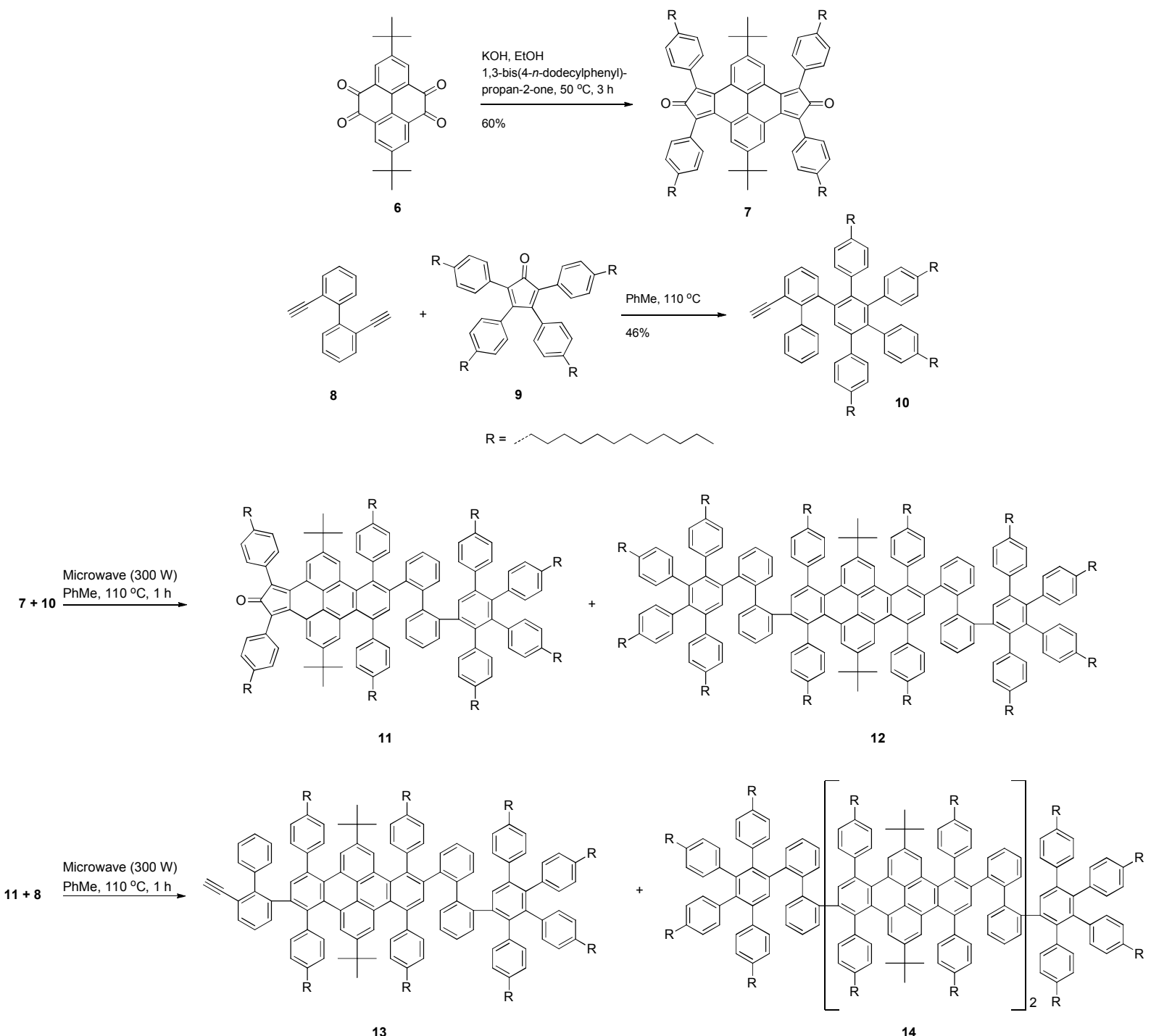

Scheme 5. Microwave assisted Diels-Alder protocol used by Müllen and co-workers ${ }^{42}$ for the synthesis of the first two polyphenylene ribbons 12 and 14. 


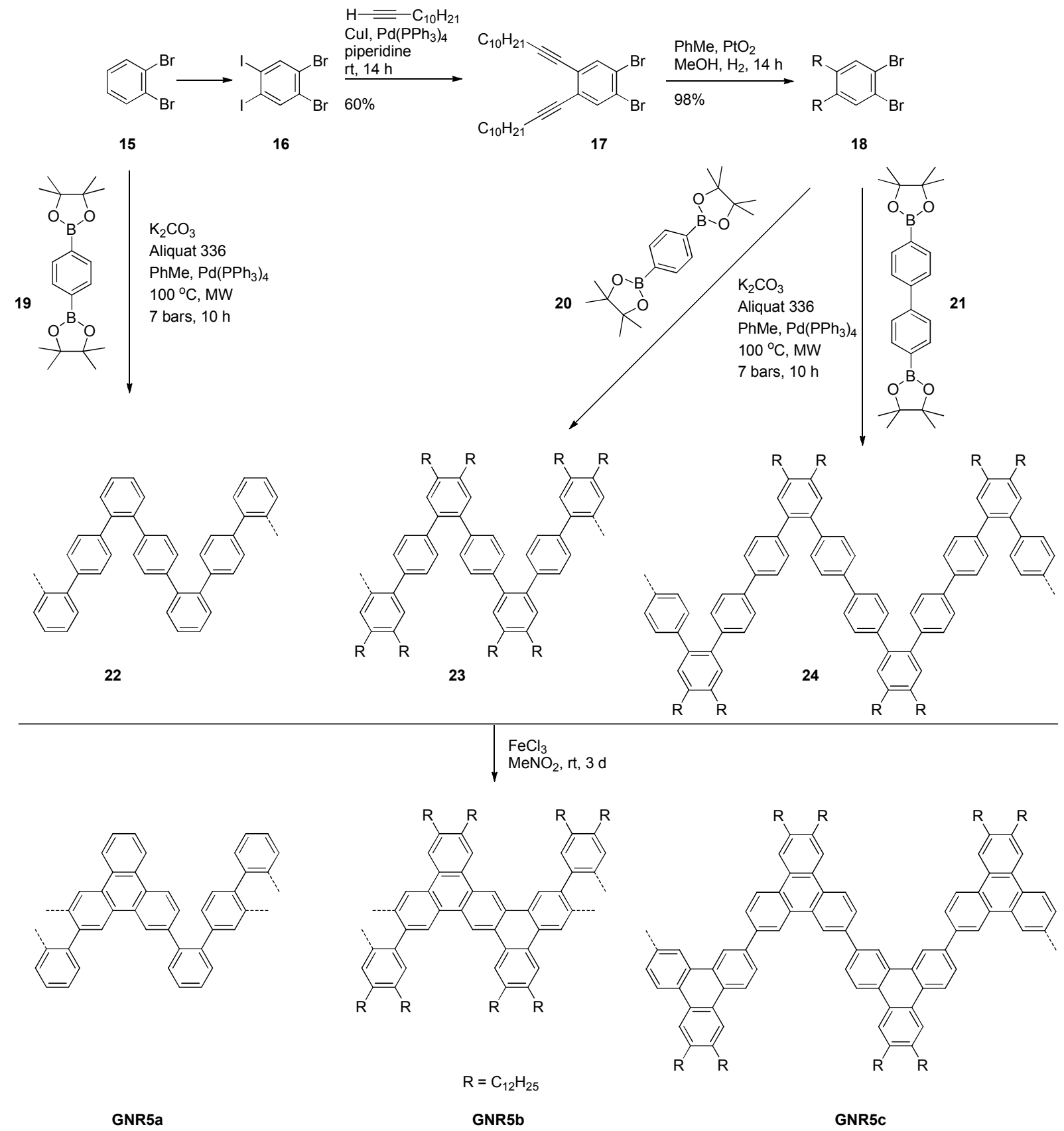

Scheme 6. Microwave assisted Suzuki-Miyaura coupling polymerization followed by Scholl cyclodehydrogenation for the production of GNRs5a-c type, with limited rigidity in the backbone. ${ }^{43}$

In 2012, Müllen and co-workers ${ }^{44}$ reported the preparation of GNRs that had broad light absorption extending up to the near-infrared region, with an optical band gap as low as $1.12 \mathrm{eV}$. The synthesis of such conductive materials is critical for most optoelectronic applications (e.g., 
photovoltaics, LEDs, optical switching devices), given that a low band gap generally enhances the charge injection/mobility towards the device electrodes. In this case, the research group began by synthesizing a para-terphenyl-based oligophenylene precursor (compound 27, Scheme 7). As shown in Scheme 7, this precursor was synthesized via a Sonogashira-Hagihara coupling reaction between a diiodo-modified terphenylene monomer (compound 25, Scheme 7) and trimethylsilylacetylene, followed by deprotection to yield 2,2"-diethynylterphenyl (compound 26, Scheme 7). Then, a Diels-Alder cycloaddition with a functionalized tetraphenylcyclopentadienone monomer under microwave conditions efficiently produced the paraterphenyl-based oligophenylene precursor in $85 \%$ yield. This precursor was then polymerized via an $A A$-type Yamamoto polymerization, producing a kinked polyphenylene precursor exhibiting molecular weights up to $35,000-40,000 \mathrm{~g} \mathrm{~mol}^{-1}$. Size exclusion chromatography of the isolated polymer showed a quite high polydispersity index (PDI $\approx 2.2$ ). Fractionation of the crude polymer led to two fractions with PDI values of 1.2 and 1.1, and weight-average molecular weights (MW) of 52,000 $\mathrm{g} \mathrm{mol}^{-1}$ (for GNR6a) and 7,200 $\mathrm{g} \mathrm{mol}^{-1}$ (for GNR6b), respectively. It was thus demonstrated that $A A$-type Yamamoto polymerization was preferable over the $A_{2} B_{2^{-}}$ type polymerizations, like Suzuki-Miyaura ${ }^{40,43}$ and Diels-Alder, ${ }^{42}$ that the same group had previously used for the preparation of GNRs precursors. The final synthetic step, Scholl cyclodehydrogenation of the two polymer precursors using $\mathrm{FeCl}_{3}$ as oxidant in a mixture of dichloromethane and nitromethane, afforded the two target GNRs (Scheme 7, GNR6a-b).
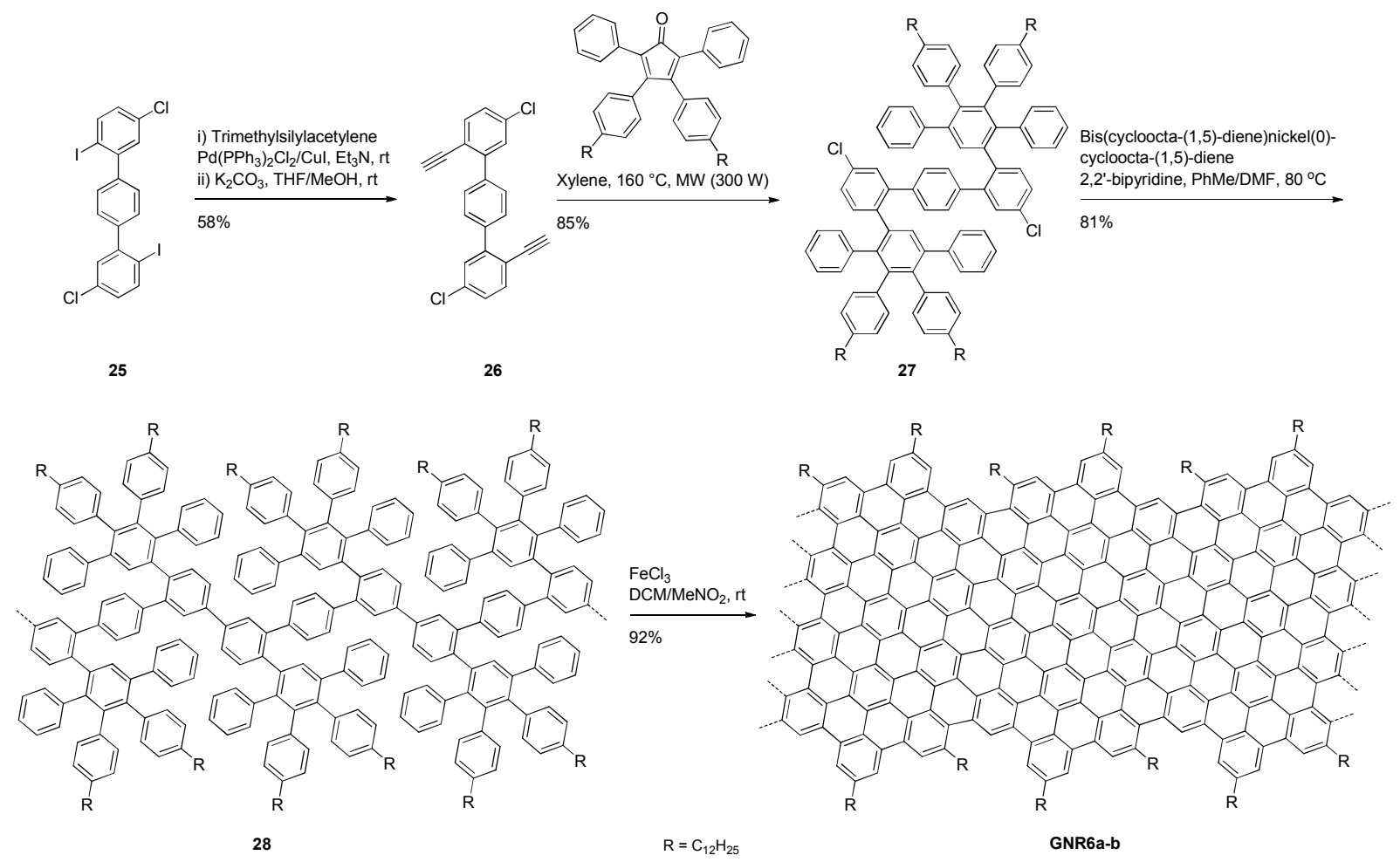

Scheme 7. Synthesis of GNR6a-b exhibiting optical band gaps as low as $1.12 \mathrm{eV} .^{44}$ 
UV/Vis studies of the as-obtained GNRs (6a and 6b), using NMP as solvent, revealed that the absorption edges extend to 1109 and $812 \mathrm{~nm}$, respectively, corresponding to optical band gaps of 1.12 and $1.53 \mathrm{eV}$, respectively.

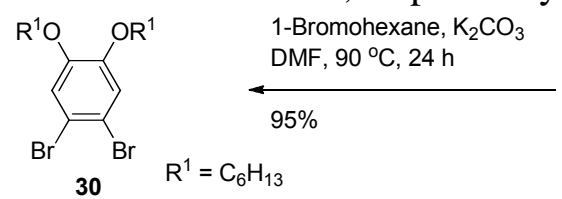

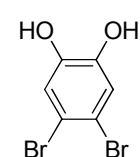

29
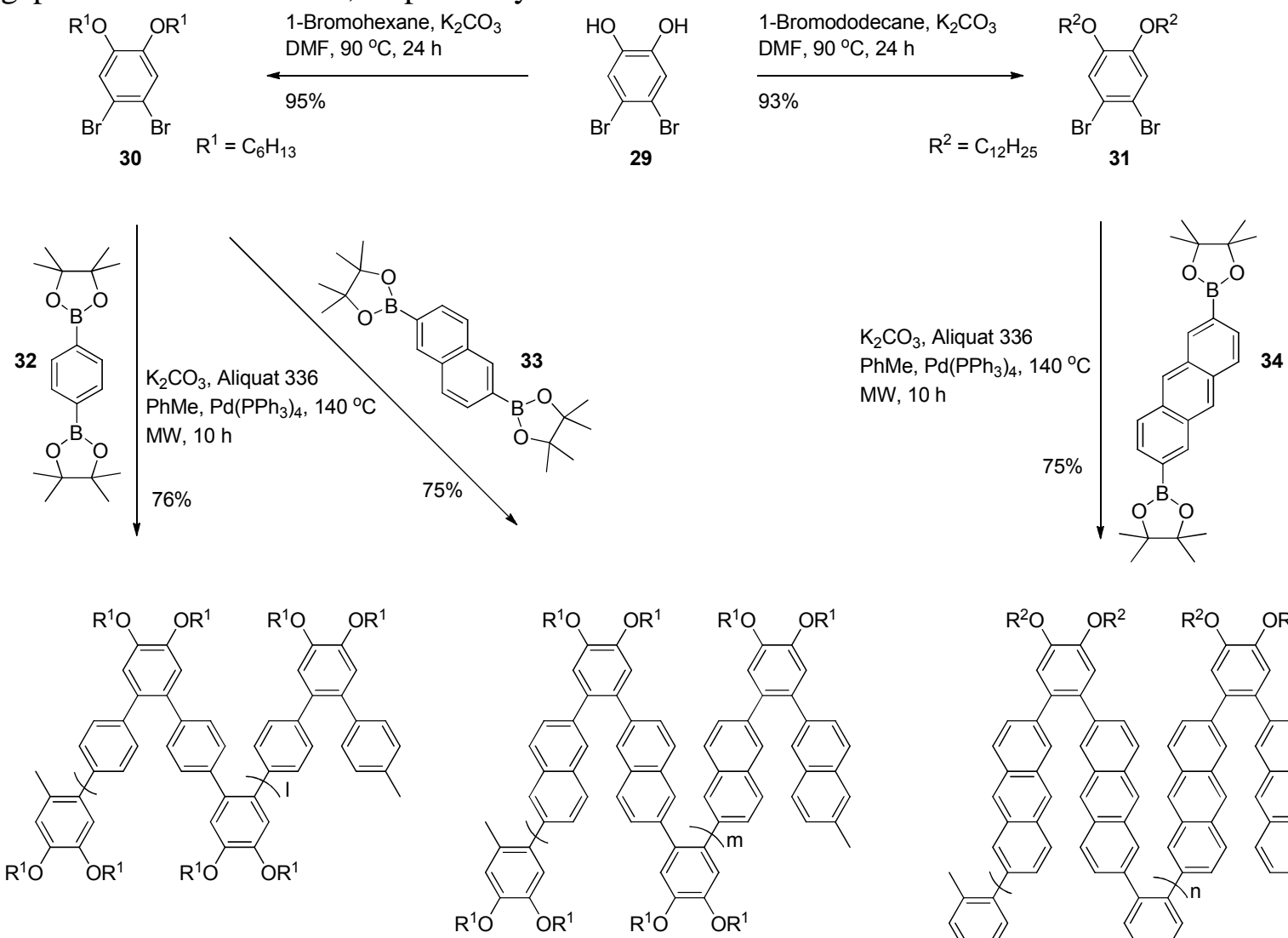

35

36

Poly-Ph $(76 \%)$

Poly-Np $(75 \%)$

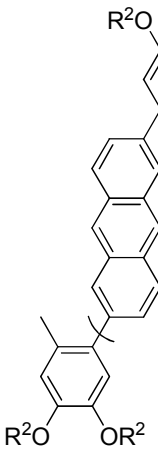

$\mathrm{R}^{2}$

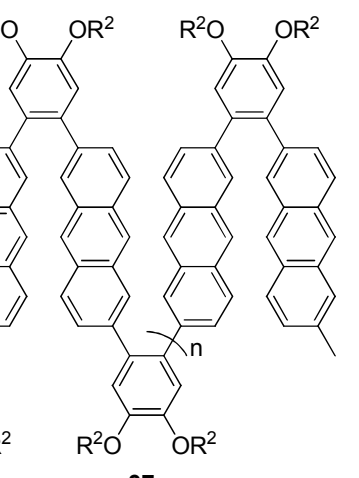

37

Poly-An (75\%)

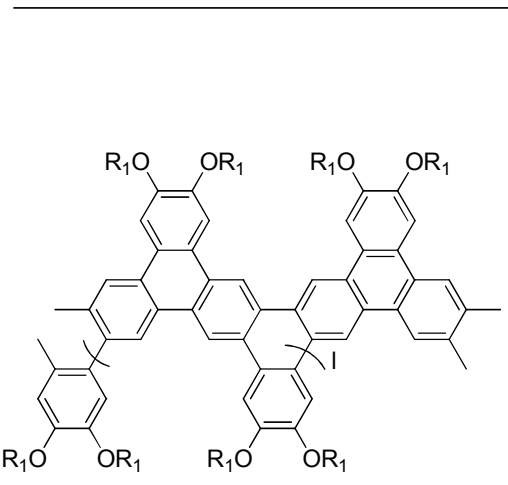

GNR-Ph (83\%)

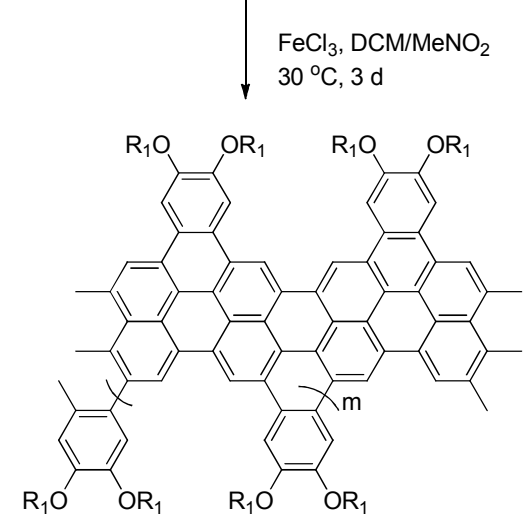

GNR-Np $(42 \%)$

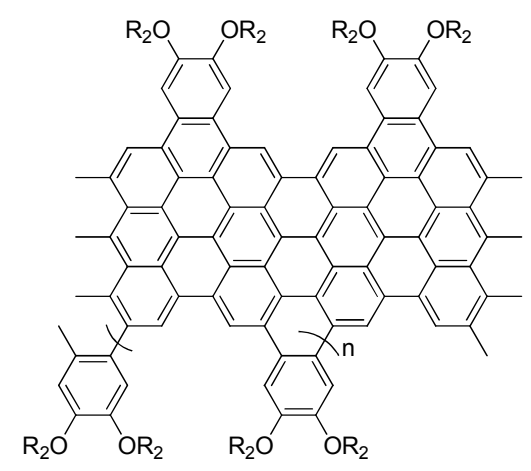

GNR-An (20\%)

Scheme 8. Synthesis of GNR-Ph, GNR-Np, and GNR-An by Kim, Jung and Jo. ${ }^{45}$ 
One year later, Kim Jung and Jo published the synthesis of GNR precursor polymers containing phenylene, naphthalene, or anthracene units for the fabrication of GNRs (Scheme 8), by using the Suzuki polymerization method developed by the group of Müllen. ${ }^{43}$ The respective polymer precursors, named on the basis of the monomer employed as Poly-Ph (phenylene), Poly$\mathrm{Np}$ (naphthalene), or Poly-An (anthracene), were then converted into the corresponding GNRs (GNR-Ph, GNR-Np and GNR-An) by Scholl cyclodehydrogenation using $\mathrm{FeCl}_{3}$ as oxidant. The degree of cyclodehydrogenation was studied via ${ }^{1} \mathrm{H}$ NMR spectroscopy and was found to be $75 \%$ for GNR-An, $78 \%$ for GNR-Np, and 100\% for GNR-Ph. Moreover, completely cyclodehydrogenated GNR-Np and GNR-An were insoluble in organic solvents, while completely cyclodehydrogenated GNR-Ph was soluble in most organic solvents. All GNR films obtained by the respective materials were applied in thin film transistor devices (TFTs), where they showed ambipolar charge transport behavior. The GNR film prepared from the anthracene-based GNRs exhibited the highest TFT performance (a hole mobility of $3.25 \times 10^{-2} \mathrm{~cm}^{2} / \mathrm{V} \mathrm{s}$ and an electron mobility of $7.11 \times 10^{-3} \mathrm{~cm}^{2} / \mathrm{V} \mathrm{s}$ ), owing to its more extensive conjugation and larger nanoribbon width, compared to the GNRs prepared from the phenylene and naphthalene-based polymers.

In the same year, Kubo and co-workers ${ }^{46}$ published a study on the synthesis and characterization of quarteranthenes (Scheme 9, GNR7). Quarteranthenes are small GNRs produced by reductive aromatization and consequent cyclization of specially modified precursor molecules. The electronic absorption spectra of the quarteranthene with four tert-butyl groups appended around the core (Scheme 9), exhibit a low-energy band centered at $917 \mathrm{~nm}$ with a long tail that extends up to $1300 \mathrm{~nm}$ (infrared). Absorption decay measurements showed that this specific quarteranthene when exposed to air under ambient light has a half-life of only $15 \mathrm{~h}$ at room temperature. Studies on the molecular geometry, magnetic and optical properties, as well as the chemical reactivity at the zigzag edges of quarteranthenes led to the conclusion that the physical and chemical properties of these molecules are explained very well by the edge localization of the unpaired electrons. By observing the room temperature ferromagnetic correlation via SQUID measurements, quarteranthenes can be considered as the minimal structural element of zigzag GNRs that still shows peculiar magnetic properties. Moreover, the multispin correlation in zigzag GNRs, which is antiferromagnetic across the ribbon main axis and ferromagnetic along the ribbon's main axis, could be simulated by aligning anthenes sideby-side. 


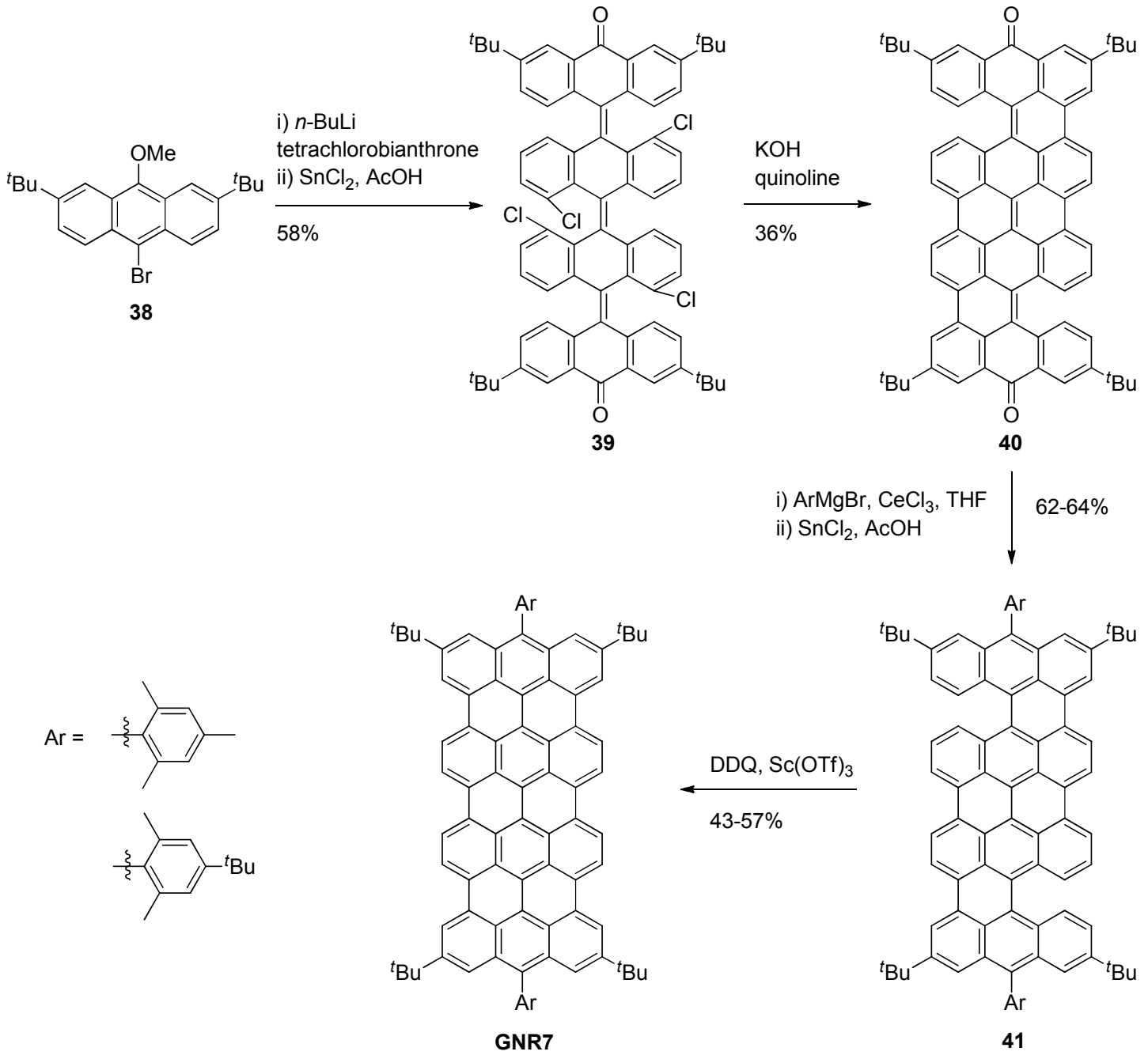

Scheme 9. Synthetic route to quarteranthenes (GNR7). ${ }^{46}$

In 2014, Sinitskii and coleagues ${ }^{37}$ reported the bottom-up, large scale (gram quantities) synthesis of high-aspect-ratio GNRs that are only $\sim 1 \mathrm{~nm}$ wide and have atomically smooth armchair edges (Scheme 10, compound 46a, GNR8a). These GNRs were produced via a Yamamoto coupling of the appropriate molecular precursors (compound 45a and 46a, Scheme 10), followed by a $\mathrm{FeCl}_{3}$ mediated Scholl cyclodehydrogenation. The as-obtained GNRs exhibit a large electronic bandgap of $\sim 1.3 \mathrm{eV}$, which was significantly higher than any value reported in experimental studies of graphene nanoribbons prepared by top-down approaches. These synthetic GNRs can have lengths higher than $100 \mathrm{~nm}$ and self-assemble in highly-ordered fewmicrometer-long 'nanobelts' that can be visualized by conventional microscopy techniques, and potentially used for the fabrication of electronic devices. Atomic force microscopy (AFM) images determined the height of the GNR nanobelts produced to be $\sim 3 \AA$, which is very close to the interlayer spacing in graphite (3.35 $\AA$ ). Height profiles of different AFM images for 480 such 
nanobelts were no more that $\sim 5 \AA$, suggesting that these nanobelts were not GNRs stacks since their thickness corresponded to the thickness of a single graphene ribbon. SEM imaging, used to determine the widths of the same GNR nanobelts, indicated widths of a few nm up to about 60 $\mathrm{nm}$ : the narrowest GNR nanobelt was calcuated to be about $10 \mathrm{~nm}$ wide, which corresponds to 5 to 6 GNRs arranged side by side. Additionally, STM images revealed GNRs that were at least $100 \mathrm{~nm}$ long. These GNRs could be used to bridge electrodes fabricated by standard electronbeam lithography (EBL) techniques and be applied in technological fields like field effect transistors, logic gates, and photovoltaic devices.

In a parallel work, Sinitskii and co-workers ${ }^{38}$ achieved the bottom-up, large scale synthesis of narrow, nitrogen-doped graphene nanoribbons (N-GNRs) via the same method but by changing only one step of the synthesis (Scheme 10, compound 46b, GNR8b). The monomer molecule used as precursor for the N-GNR synthesis was not symmetric relative to the plane perpendicular to the coupling direction. As a result, the positions of nitrogen atoms in the final N-GNR edge may vary. However, the N/C ratio of $1 / 20$ in the ribbon produced should be the same regardless of how the monomer molecules couple with each other. STM and AFM images acquired after depositing the N-GNRs on mica and mica-(Au111) substrates, revealed images of nanostructures defined as "GNR nanobelts", 37

STM images of the N-GNR nanobelt on $\mathrm{Au}(111)$ substrate illustrate a side-by-side arrangement of individual ribbons that are over $40 \mathrm{~nm}$ long. Nevertheless, it was unclear if these nanobelts existed in solution or formed on the substrate by capillary forces during the solvent evaporation. Transmission electron microscopy (TEM) revealed the N-GNR nanobelts to be 8 to $20 \mathrm{~nm}$ wide and up to $1 \mathrm{~mm}$ long, corresponding to 8 to 12 of the as-produced N-GNRs arranged in a side-by-side fashion.

In 2014, Müllen and co-workers ${ }^{47}$ reported the bottom-up synthesis of structurally welldefined liquid phase-processable GNRs via their Diels-Alder polymerization strategy. ${ }^{42}$ More specifically, non-planar polyphenylene precursors were synthesized (compound $\mathbf{5 1}$, Scheme 11 and compound 57, Scheme 12) and then "planarized" to afford two types of GNRs, having dodecyl or branched 2-decyltetradecyl chains, respectively, for increased solubility/proccessability (Schemes 11-GNR9 and 12-GNR10). The GNR9 polymeric precursor (compound 52, Scheme 11) exhibited molecular weights one order of magnitude greater than those obtained via the Yamamoto coupling polymerization previously published by the same team, ${ }^{44}$ although the polydispersity index was quite high (between 3 and 14), which was attributed to the possible formation of cyclic oligomers in addition to linear polymers. After fractionation, dynamic lightscattering experiments showed that the absolute MW value of the GNR9 polymeric precursor 52 was about $470 \pm 30 \mathrm{~kg} \mathrm{~mol}^{-1}$, with a PDI value between 1.7 and 1.9. Similarly, for the GNR10 polymeric precursor (compound 58, Scheme 12), the MW values varied from $160 \pm 20$ to $370 \pm 40$ $\mathrm{kg} \mathrm{mol}^{-1}$ and the PDI values were between 3.4 and 4.9, respectively. 


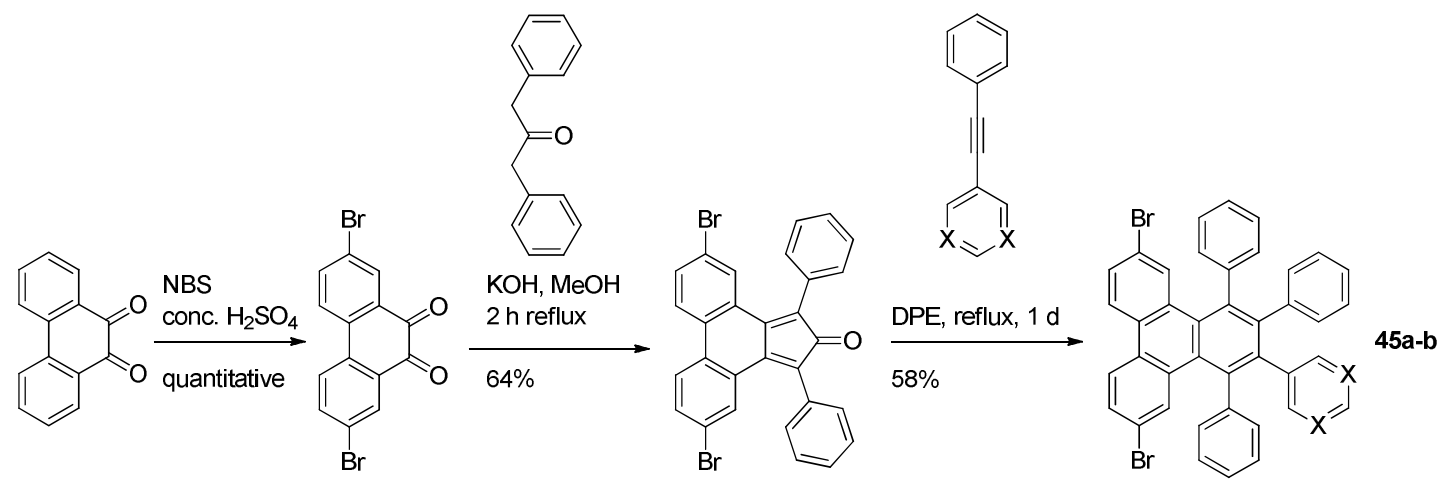

42

43

44

$\mathrm{X}=\mathrm{H}=45 \mathrm{a} / 46 \mathrm{a}, \mathrm{GNR8} \mathrm{a}$

$X=N=45 b / 46 b$, GNR8b

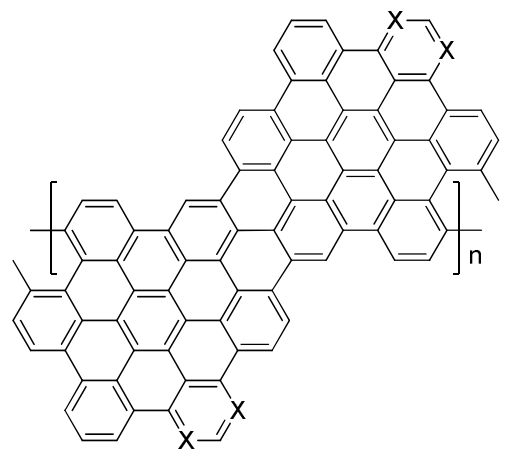

GNR8a-b
$\mathrm{FeCl}_{3}$

$\mathrm{DCM} / \mathrm{MeNO} \mathrm{N}_{2}$

$\stackrel{\mathrm{rt}, 1 \mathrm{~d}}{4}$

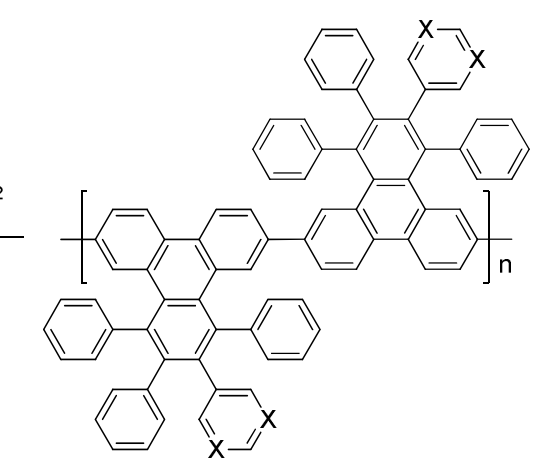

46a-b

Scheme 10. Sinitskii large scale synthesis of GNRs (GNR8a) and N-GNRs (GNR8b). ${ }^{37,38}$

STM images of GNR9 (Scheme 11) on highly oriented pyrolytic graphite (HOPG) showed a well-organized self-assembled monolayer of straight and uniform nanoribbons of up to about 60 $\mathrm{nm}$ in length. AFM phase imaging of GNR10 (Scheme 12) on HOPG similarly showed a highly organized self-assembled monolayer of straight and uniform nanoribbons, but with lengths exceeding $200 \mathrm{~nm}$. Moreover, the as-obtained GNRs had large optical bandgaps of $1.88 \mathrm{eV}$, while non-contact time-resolved terahertz conductivity measurements revealed excellent chargecarrier mobility within individual GNRs. 


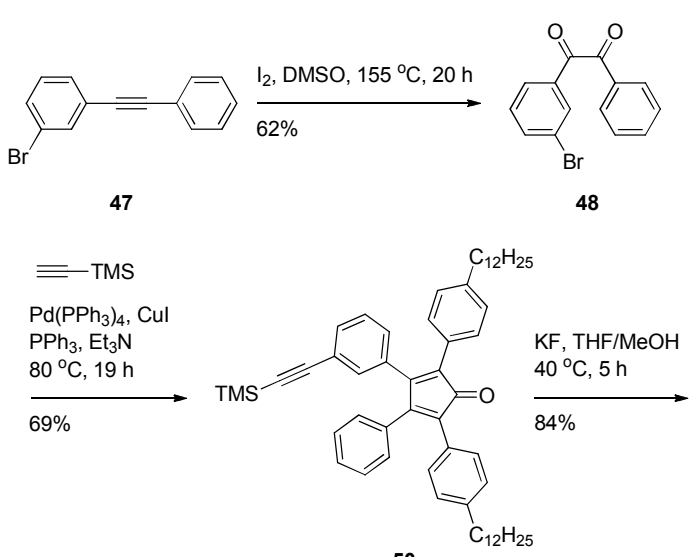

50

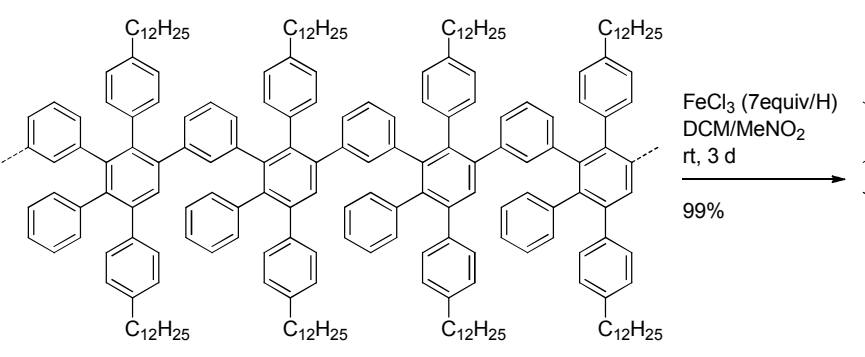

52
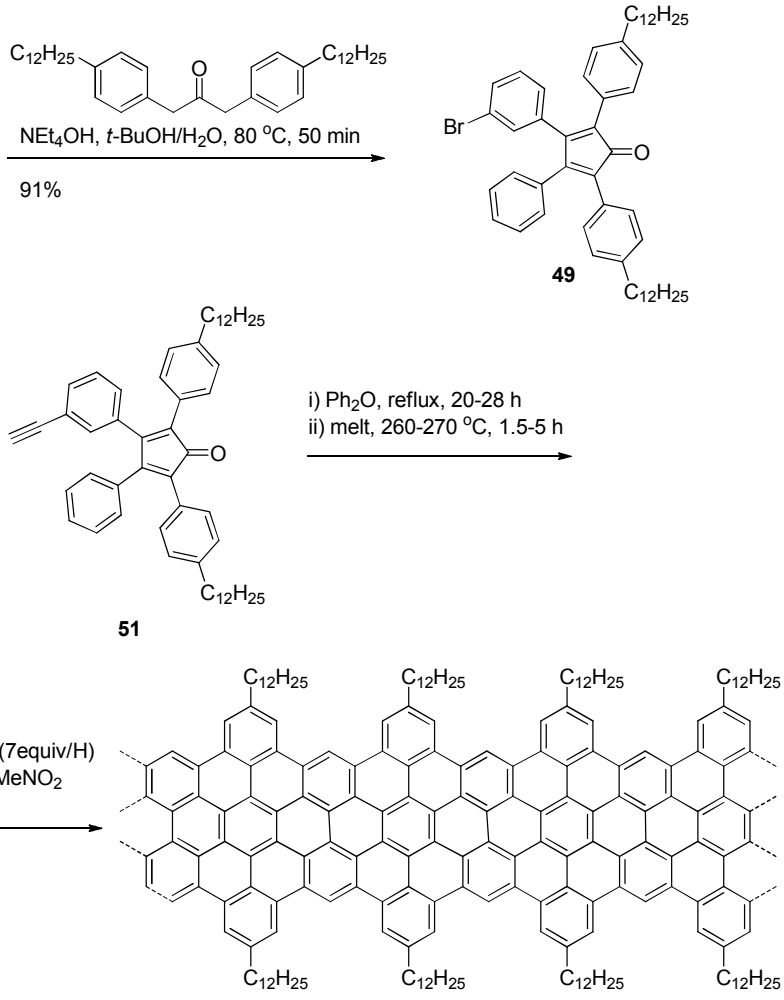

GNR9

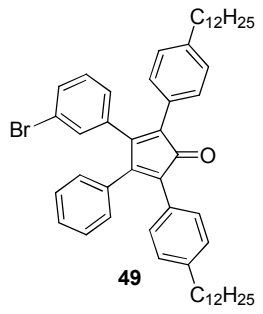

i) $\mathrm{Ph}_{2} \mathrm{O}$, reflux, 20-28 h

ii) melt, $260-270^{\circ} \mathrm{C}, 1.5-5 \mathrm{~h}$

Scheme 11. Müllen's synthesis of dodecyl-decorated GNR9. ${ }^{47}$

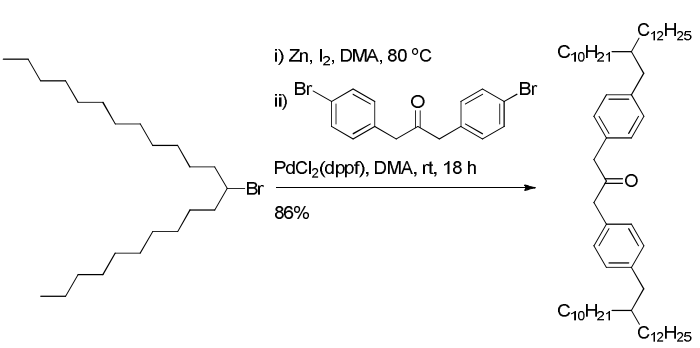

53

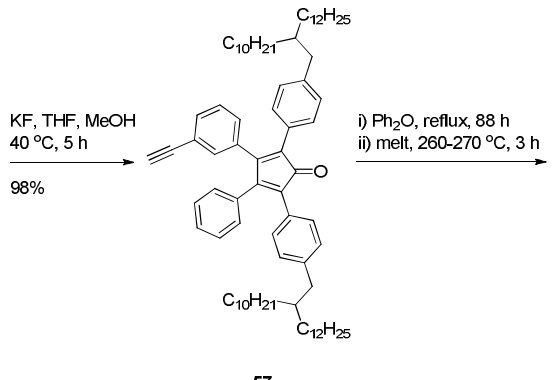

57

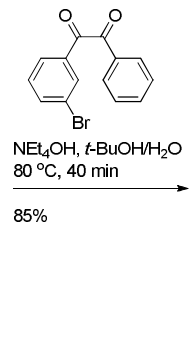

54

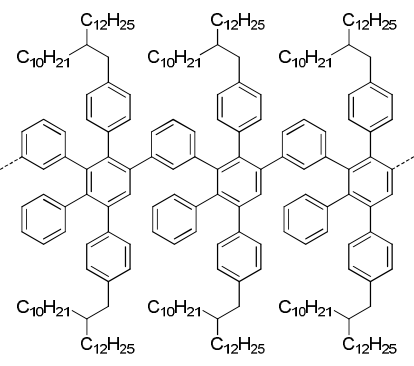

58

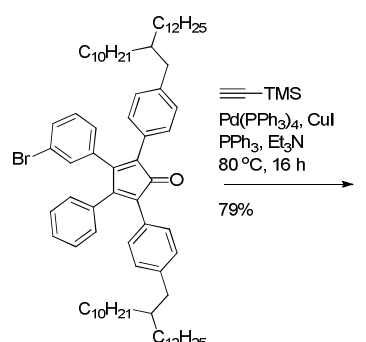

55

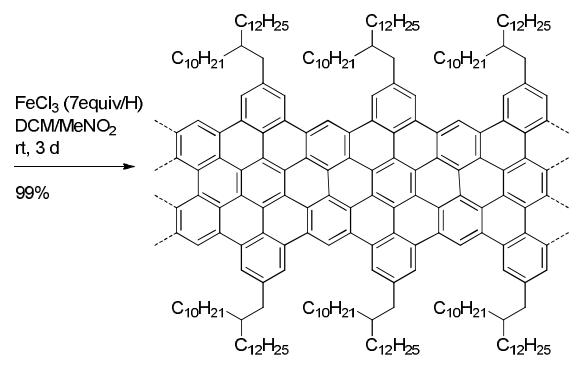

GNR10 


\subsection{GNRs synthesis via the conversion of precursors inside CNTs}

In 2011, Khlobystov and co-workers ${ }^{48}$ reported an alternative method for the preparation of a sulfur-terminated graphene nanoribbon within a single-walled carbon nanotube (SWCNT). The basic idea for this strategy came from fullerenes, close relatives of GNRs, that can undergo chemical transformations inside SWNTs, triggered by heat or electron beam radiation (e-beam). In this setup, the carbon nanotube is not involved in the reactions of the guest-molecules, but imposes desired spatial limitations on the structure of the product. For example, fullerene epoxide $\left(\mathrm{C}_{60} \mathrm{O}\right)$ can form strictly linear polymer chains within SWNTs, while the same reaction leads to the formation of branched, highly convoluted polymers, when performed outside the nanotube. ${ }^{53}$ On this basis, SWCNTs were used as efficient templates/hosts for the self-assembled preparation of GNRs. As GNR precursor monomers the researchers used either modified fullerenes $\{N$-methyl-2-[4-(liponyloxy)benzyl]-[5,6]-Sc $3 \mathrm{~N} @ \mathrm{C} 80$ fulleropyrrolidine $\}$ or tetrathiafulvalene/ $\mathrm{C}_{60}$ mixtures. Electron microscopy of the products revealed elliptical distortion of the nanotube, as well as helical twist and screw like motion of the nanoribbon. The authors suggest that these effects create new ways to control the properties of these nanomaterials, such as the electronic band gap and the concentration of charge carriers. The overall procedure of producing GNRs inside SWCNTs was dominated by two factors: i) 1-D confinement at the nanoscale ensures propagation of the GNR in only one dimension; and ii) the incorporation of heteroatoms into the predominantly carbon-based elemental feedstock leads to the termination of dangling bonds, stabilizing the structure thermodynamically. The semiconducting properties of sulfur GNRs and their dynamic behavior inside the nanotube (rotation and translation), may pave the way for future applications of these materials in electronic and mechanical nanodevices.

In the same year, Talyzin, Anoshkin and co-workers ${ }^{49}$ also reported the synthesis of GNRs encapsulated in SWCNTs. In this case, GNRs were hydrogen-terminated, by using confined polymerization and fusion of polycyclic aromatic hydrocarbon (PAH) molecules, like coroneneand perylene (compounds 59 and 60, Scheme 13). More specifically, SWNTs opened by oxidation treatment, were exposed to coronene or perylene vapors in reactors sealed with argon at ambient pressure. Annealing was then performed for 1 to $24 \mathrm{~h}$ starting from just above the melting point of coronene and up to the temperatures at which bulk coronene fusion reactions were observed. Similar experiments were carried out for the case of perylene. SWNTs served as nucleation centers for coronene or perylene fusion, catalyzing the reaction and leading to the formation of bulk hydrocarbon products on the outer side of SWNTs and GNRs inside the SWNTs. Therefore, the aligning effect of one confined space inside the nanotubes on the orientation of the encapsulated coronene or perylene molecules was crucial for the formation of the nanoribbons. Scheme 13 shows the proposed structures of the nanoribbons formed, by using either coronene (GNR11a-b) or perylene (GNR12) precursors. HRTEM microscopy of the asproduced GNRs revealed that these were formed inside the SWNTs with almost $100 \%$ filling, and that their respective length was limited only by the length of the nanotubes used in the study. Moreover, the nanoribbons were non-uniform in their width, with a visible variation in the range of about 0.5 to $1.0 \mathrm{~nm}$ (the respective diameter of the nanotubes used were between 1.7 and 2.0 
$\mathrm{nm})$. The maximum width of the GNRs was limited by the diameter of the SWNTs, while their geometrical shape was more complex. Additionally, the obtained GNRs 11 and 12 twisted and frequently formed helical configurations. Finally, the authors suggest that various combinations of nanotubes and nanoribbons (i.e. metallic GNRs inside metallic SWNTs or metallic GNRs inside semiconducting SWNT) hold promise for the synthesis and application of new materials with unusual properties.

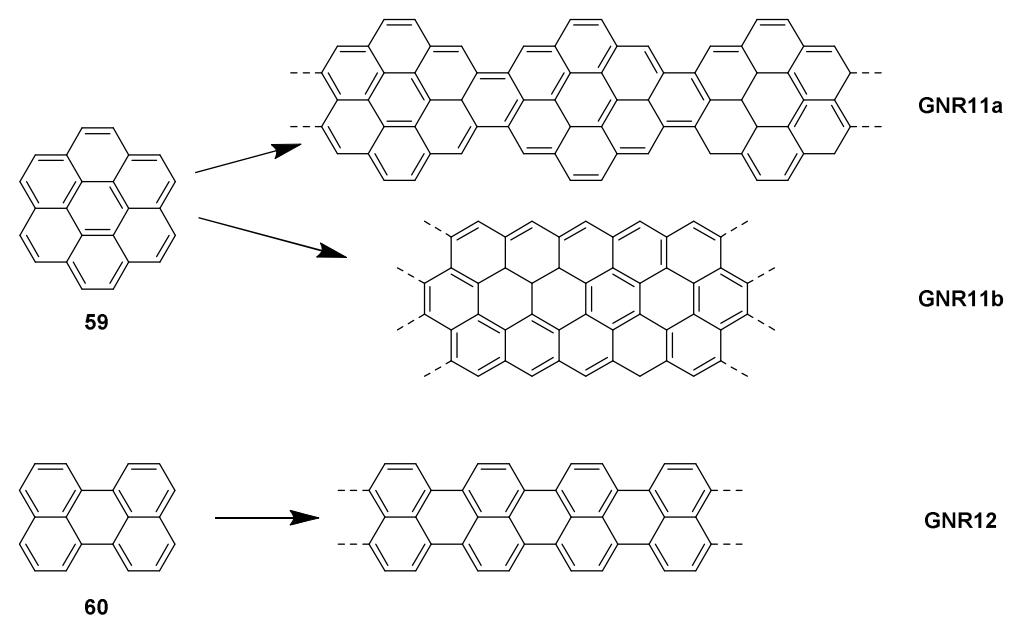

Scheme 13. The suggested structures of hydrogen terminated GNRs formed inside SWCNTs by using either coronene (GNR11) or perylene (GNR12) precursors. ${ }^{49}$

\subsection{GNRs synthesis through surface-assisted polymerization}

Given that graphene nanoribbons consist of extended aromatic systems, their synthesis, characterization, purification, and proccessability all suffer from the strong intermolecular $\pi$ - $\pi$ stacking interactions that leads to poor solubility in conventional organic solvents. One way to overcome these obstacles, is to employ covalent synthesis on surfaces. In this approach, molecular layers of oligophenylene precursors are deposited on a suitable metallic surface and then subjected to thermal-assisted polymerization and cyclodehydrogenation, eventually producing the desired graphene.

In 2010, Cai and co-workers ${ }^{50}$ published a study on the atom-precise bottom-up fabrication of straight- and chevron-type GNRs having various topologies and widths, by employing a surface-assisted coupling of molecular precursors followed by cyclodehydrogenation. The topology, width, and edge periphery of the graphene nanoribbons obtained were defined by the structure of the precursor monomers, which could be designed to give access to a wide range of different GNRs. Scheme 14 shows the synthesis of these GNRs, by using various dibrominated precursor monomers based on bisanthracenes (compound 61, GNR13), polyphenylenes (compound 45, GNR14), or a combination of the latter with triiodo-substituted polyphenylenes (compounds 45 and 46, GNR15), over an $\mathrm{Au}(111)$ surface. The first step, in the fabrication of the as-prepared GNRs, is to thermally activate the precursor molecules. For GNR13 (Scheme 14), 
annealing at $200{ }^{\circ} \mathrm{C}$ imparts the dehalogenated intermediates with enough thermal energy to diffuse along the surface of $\mathrm{Au}(111)$ and form single covalent $\mathrm{C}-\mathrm{C}$ bonds between each monomer, thus producing the respective polymer chains. For GNR14 and GNR15, the annealing temperature was set at $250{ }^{\circ} \mathrm{C}$. GNR13 is eventually obtained after a second annealing step at $400{ }^{\circ} \mathrm{C}$, which induced intramolecular cyclodehydrogenation of the polymer chain and hence the formation of an armchair-edged ribbon with half the periodicity of the mother polymeric chain.

In a similar manner, GNR14 and GNR15, were produced with the second annealing step set at $440{ }^{\circ} \mathrm{C}$. GNR13 and GNR14 both exhibited a band gap of $1.6 \mathrm{eV}$, despite their differences in terms of topology. Moreover, this method for the fabrication of GNR13 and GNR14 also worked well when the $\operatorname{Au}(111)$ template was replaced by $\operatorname{Ag}(111)$, as shown from STM images of straight- and chevron-type GNRs grown on $\mathrm{Ag}(111)$ surfaces. Additionally, when the precursor monomers for GNR13 and GNR14 were deposited together on the substrate surface and thermally activated, the homomolecular coupling reaction was highly selective over the more sterically hindered heteromolecular one. Finally, the strategy followed for the preparation of GNR15 should also enable the controlled growth of various GNR heterojunctions.

In 2013, Bronner and co-workers ${ }^{51}$ expanded the work of $\mathrm{Cai}^{50}$ by preparing analogous, nitrogen-doped GNRs via the polymerization of specially-designed monomers on an Au(111) surface. Moreover, they studied the band gap of these nitrogen-doped GNRs with surfacesensitive electron spectroscopies. As shown in Scheme 15, they incorporated one or two nitrogen atoms in the parent monomer (monomers $\mathbf{6 5}$ and 66, respectively). These monomers were used to generate GNR16 and GNR17, respectively, with different nitrogen doping levels leading to different electronic properties. The synthesis of the new monomers 65 and 66 was accomplished via Diels-Alder reactions of the appropriate cyclopentadienone (compound 44, Scheme 15) with either mixed phenylpyridyl-acetylene or bispyridylacetylene, followed by immediate cheletropic $\mathrm{CO}$ extrusion. By using this approach, graphene nanoribbons were successfully doped in a predefined manner by selective nitrogen substitution of the precursor monomers, a modification that does not interfere with their on-surface chemical reactivity. Angle-resolved high-resolution electron energy loss spectroscopy (HREELS) in combination with photoelectron spectroscopy of these GNRs showed that the band gap was linearly shifted in relation to the electronic structure of the environment of the GNR, but remained almost unchanged in magnitude, as expected for a pyridine-like $s p^{2}$ nitrogen at the edges of armchair GNRs. Thus, the magnitude of the band gap of the nitrogen monosubstituted GNR16 remains unchanged, compared with that of the analogous pristine GNR of Cai, while the band gap of the nitrogen disubstituted GNR17 was reduced by $3 \%$. 


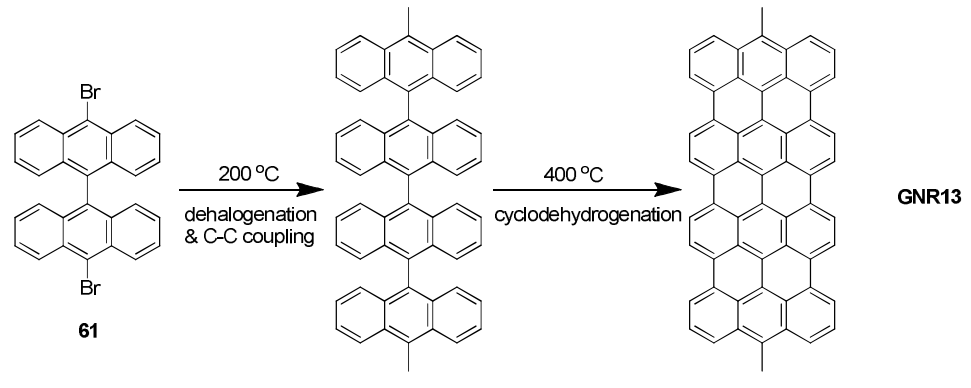

62

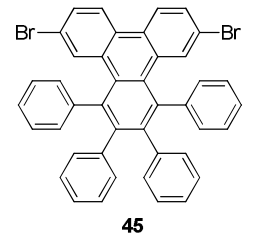

45

$250^{\circ} \mathrm{C}$

dehalogenation \& C-C coupling
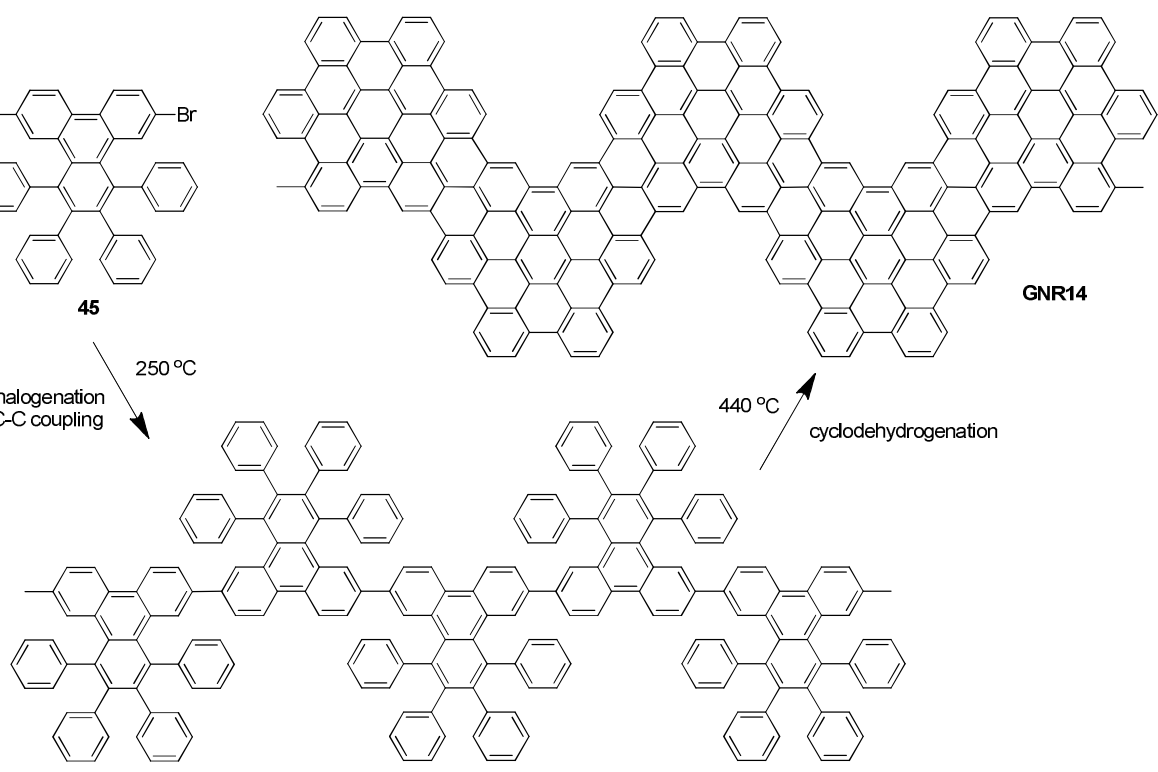

64
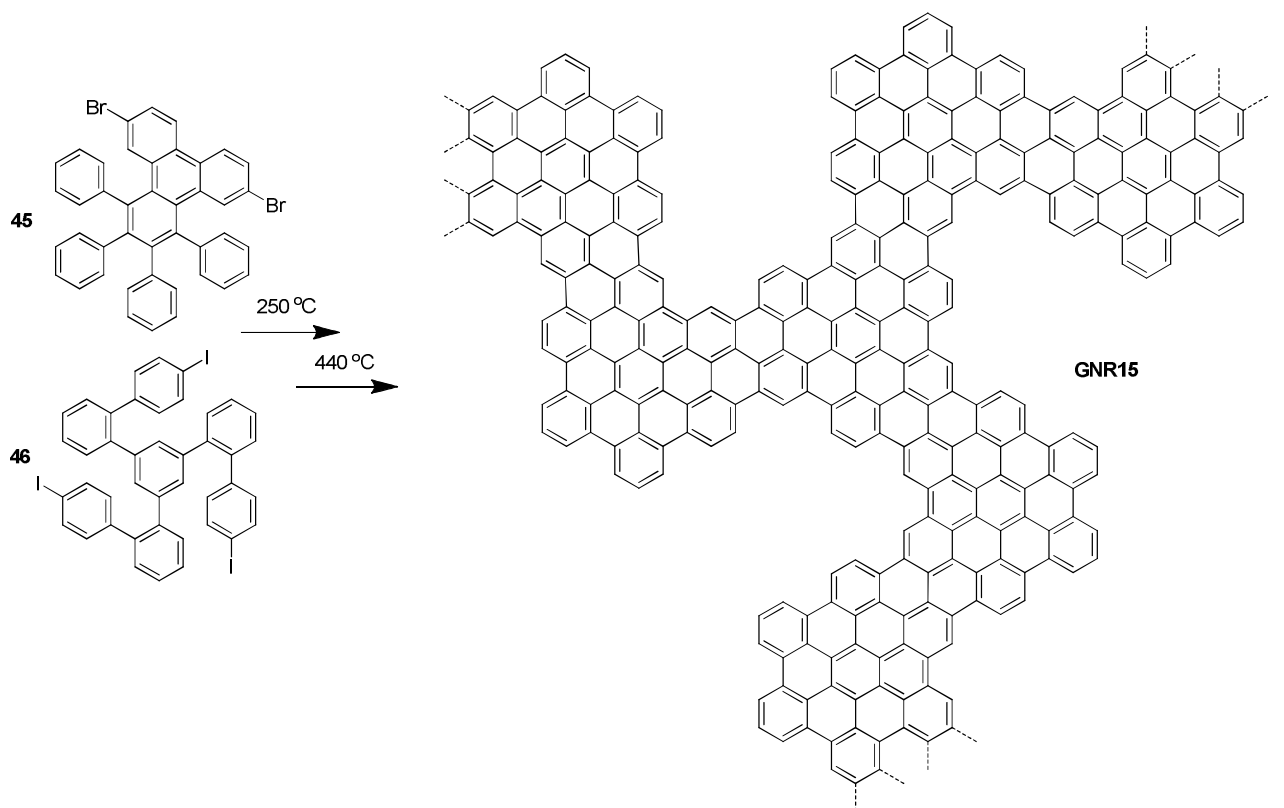

Scheme 14. Surface-assisted production of straight-type (GNR13), chevron-type (GNR14), and junction-type (GNR15) GNRs, by using properly designed precursor molecules. ${ }^{50}$ 

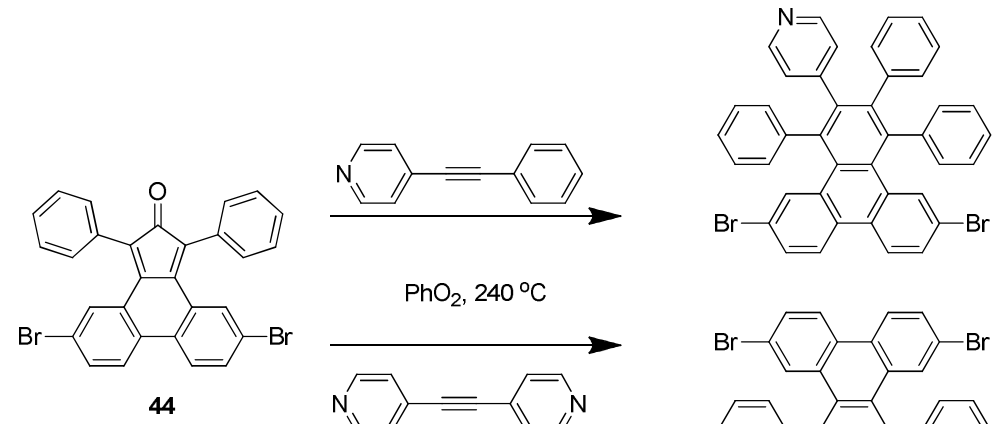

$65(48 \%)$
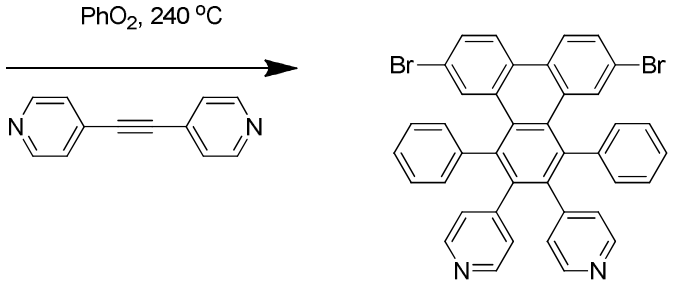

$66(62 \%)$
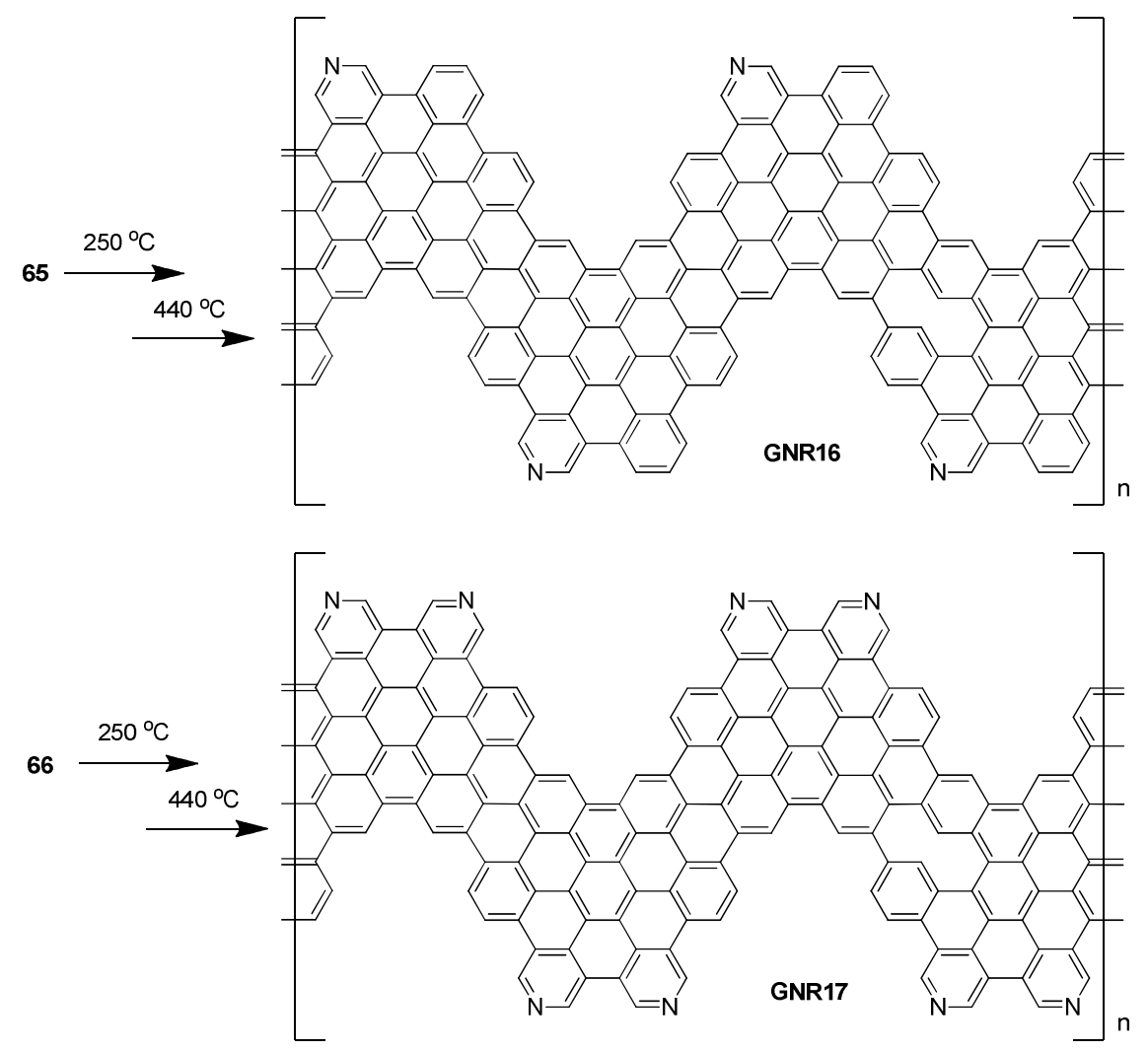

Scheme 15. Surface-assisted preparation of N-doped chevron-type GNRs 16 and $17 .{ }^{51}$

One year later, Sakaguchi, Nakae and co-workers" took the "surface-assisted polymerization" approach one step further. They demonstrated the large-scale growth of armchair-edged GNRs on $\mathrm{Au}(111)$ surfaces in extremely low-vacuum conditions. To accomplish that, they introduced a new method based on radical-polymerized chemical vapor deposition (RP-CVD). By using bromo-substituted precursor monomers based on perylene (compounds 67a and 67b), anthracene (compound 61), and tetracene (compound 68), as shown in Scheme 16 respectively, armchair-edged GNRs 2, 3, or 4 benzene rings wide were produced by this 
technique (Scheme 16, GNRs 18-20). The experimental procedure begins by placing the appropriate monomers in a quartz boat, which are then evaporated at $200-250{ }^{\circ} \mathrm{C}$. The vapors pass through the hot walls of a quartz tube and subsequently deposit onto the substrate at 250$300{ }^{\circ} \mathrm{C}$ for $15 \mathrm{~min}$ (to form the GNR prepolymers by radical-polymerization). The temperature was then raised to $400-450{ }^{\circ} \mathrm{C}$ and maintained there for $10 \mathrm{~min}$ to dehydrogenate the prepolymers to GNRs. The optical absorption spectra of the GNRs obtained vary from the violet up to the infrared region of the electromagnetic spectrum, depending on their ribbon width. The band gaps of poly(perinaphthalene) (GNR18), poly(perianthracene) (GNR19), and poly(peritetracene) (GNR20) were $0.8,1.6$ and $1.3 \mathrm{eV}$, respectively. The photoconductivity of bulk films of RP-CVD-grown GNRs was investigated via white light illumination from a light-emitting diode. GNR18 and GNR19 showed 7.3 and $4.0 \%$ of current-gain upon illumination, respectively. These values are comparable to the value of $2.7 \%$ obtained from P3HT (poly-3-hexylthiophene), which is a conventional p-type photoconductor used in photovoltaics. These results revealed once again the potential of GNRs as an excellent photoconductor.
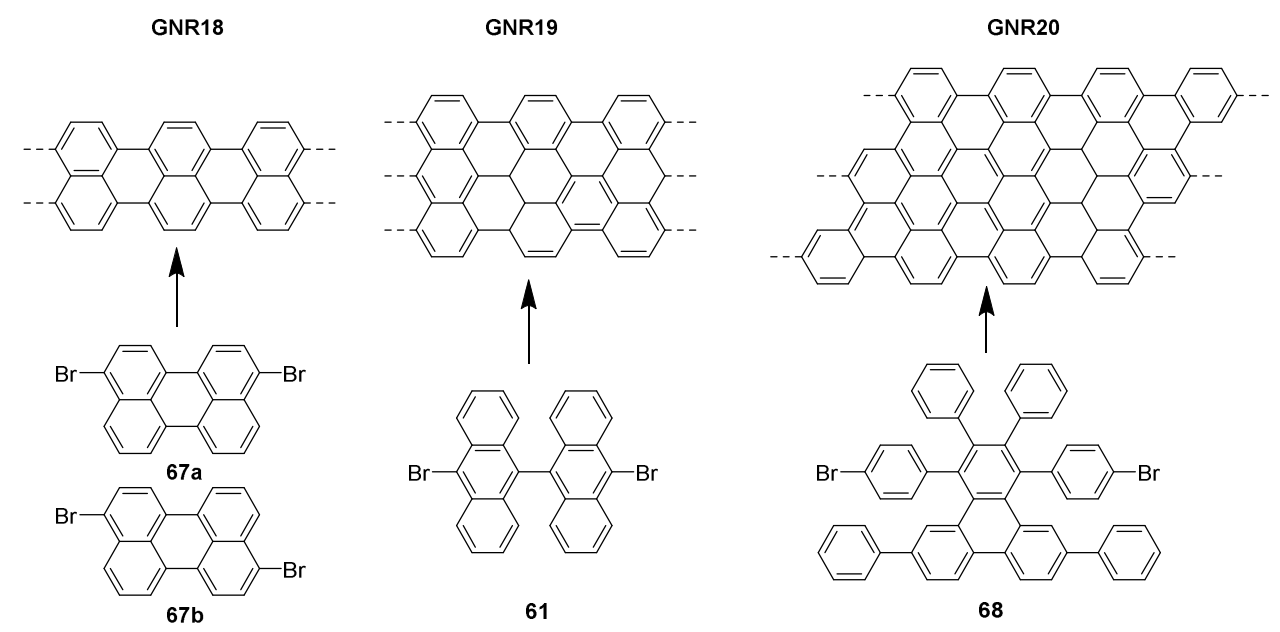

Scheme 16. RP-CVD production of GNRs 18-20, being 2, 3, or 4 benzene rings wide. ${ }^{39}$

\section{Conclusions}

Despite graphene's exceedingly high electron mobility at room temperature, its use in electronic applications is precluded owing to its zero band-gap. To overcome this problem, scientists are amongst others focusing their efforts into obtaining graphene nanoribbons (GNRs), that is, thin strips of graphene possessing a large aspect ratio. The electronic states of GNRs depend strongly on their edge structure: zigzag GNRs are metallic for all widths and also exhibit magnetic properties, whereas armchair GNRs can be either semiconducting or metallic depending on their width. To date, a variety of top-down approaches have been developed, including the sonochemical or electrochemical etching of graphene sheets, the lithographic patterning of graphene 
sheets, and the unzipping of carbon nanotubes. However, all top-down strategies suffer from significant drawbacks such as, but not limited to, complicated experimental setups, large nanoribbons widths, lack of control over the graphene edges, high fractions of defects, and low reproducibility. The alternative bottom-up approaches aim at addressing these issues by chemically synthesizing well defined, tailor-designed GNRs with predefined structural characteristics and, as a result, electronic properties. The present article summarizes and discusses all known bottom-up strategies for the synthesis of GNRs published until October 2014 and aspires to provide a useful guide for chemists and materials scientists working or interested in the field. This field has witnessed a number of significant recent advancements and many more are expected. Undoubtedly, the success of the bottom-up approaches will be of fundamental importance to the field of electronic materials and devices in general.

\section{Acknowledgements}

G.C.V. gratefully thanks Professor Michael Orfanopoulos for introducing him to the field of fullerene and physical organic chemistry, and, even more important, for being a wise mentor and a very good friend. E.K.P. thankfully acknowledges funding from an IKY Fellowship of Excellence for postgraduate studies in Greece - Siemens Program.

\section{References}

1. Kroto, H. W.; Allaf, A. W.; Balm, S. P. Chem. Rev. 1991, 91, 1213. http://dx.doi.org/10.1021/cr00006a005

2. Martín, N.; Sánchez, L.; Illescas, B.; Pérez, I. Chem. Rev. 1998, 98, 2527. http://dx.doi.org/10.1021/cr9701039

3. Vougioukalakis, G. C.; Roubelakis, M. M.; Orfanopoulos, M. Chem. Soc. Rev. 2010, 39, 817. http://dx.doi.org/10.1039/b913766a

4. Tasis, D.; Tagmatarchis, N.; Bianco, A.; Prato, M. Chem. Rev. 2006, 106, 1105. http://dx.doi.org/10.1021/cr050569o

5. Karousis, N.; Tagmatarchis, N.; Tasis, D. Chem. Rev. 2010, 110, 5366. http://dx.doi.org/10.1021/cr100018g

6. Dillon, A. C. Chem. Rev. 2010, 110, 6856. http://dx.doi.org/10.1021/cr9003314

7. Geim, A. K. Angew. Chem., Int. Ed. 2011, 50, 6966. http://dx.doi.org/10.1002/anie.201101174

8. Novoselov, K. S. Angew. Chem., Int. Ed. 2011, 50, 6986. http://dx.doi.org/10.1002/anie.201101502 
9. Chen, D.; Feng, H.; Li, J. Chem. Rev. 2012, 112, 6027. http://dx.doi.org/10.1021/cr300115g

10. Chen, L.; Hernandez, Y.; Feng, X.; Müllen, K. Angew. Chem., Int. Ed. 2012, 51, 7640. http://dx.doi.org/10.1002/anie.201201084

11. Baker, S. N.; Baker, G. A. Angew. Chem., Int. Ed. 2010, 49, 6726. http://dx.doi.org/10.1002/anie.200906623

12. Frank, E.; Steudle, L. M.; Ingildeev, D.; Spörl, J. M.; Buchmeiser, M. R. Angew. Chem., Int. Ed. 2014, 53, 5262. http://dx.doi.org/10.1002/anie.201306129

13. Trinh, C.; Kirlikovali, K. O.; Bartynski, A. N.; Tassone, C. J.; Toney, M. F.; Burkhard, G. F.; McGehee, M. D.; Djurovich, P. I.; Thompson, M. E. J. Am. Chem. Soc. 2013, 135, 11920. http://dx.doi.org/10.1021/ja4043356

14. Brabec, C. J.; Gowrisanker, S.; Halls, J. J. M.; Laird, D.; Jia, S.; Williams, S. P. Adv. Mater. 2010, 22, 3839. http://dx.doi.org/10.1002/adma.200903697

15. Hu, L.; Hecht, D. S.; Grüner, G. Chem. Rev. 2010, 110, 5790. http://dx.doi.org/10.1021/cr9002962

16. Thostenson, E. T.; Ren, Z.; Chou, T.-W. Compos. Sci. Technol. 2001, 61, 1899. http://dx.doi.org/10.1016/S0266-3538(01)00094-X

17. Han, M. Y.; Özyilmaz, B.; Zhang, Y.; Kim, P. Phys. Rev. Lett. 2007, 98, 206805. http://dx.doi.org/10.1103/PhysRevLett.98.206805

18. Li, X.; Wang, X.; Zhang, L.; Lee, S.; Dai, H. Science 2008, 319, 1229. http://dx.doi.org/10.1126/science.1150878

19. Bai, J.; Duan, X.; Huang, Y. Nano Lett. 2009, 9, 2083. http://dx.doi.org/10.1021/n1900531n

20. Novoselov, K. S.; Geim, A. K.; Morozov, S. V.; Jiang, D.; Zhang, Y.; Dubonos, S. V.; Grigorieva, I. V.; Firsov, A. A. Science 2004, 306, 666. http://dx.doi.org/10.1126/science.1102896

21. Naeemi, A.; Meindl, J. D. Electron Devic. Lett. 2007, 28, $428 . \quad$ 22. Abergel, D. S. L.; Apalkov, V.; Berashevich, J.; Ziegler, K.; Chakraborty, T. Adv. Phys. 2010, 59, 261. http://dx.doi.org/10.1080/00018732.2010.487978

22. Castro Neto, A. H.; Guinea, F.; Peres, N. M. R.; Novoselov, K. S.; Geim, A. K. Rev. Mod. Phys. 2009, 81, 109. http://dx.doi.org/10.1103/RevModPhys.81.109

23. Nakada, K.; Fujita, M.; Dresselhaus, G.; Dresselhaus, M. S. Phys. Rev. B 1996, 54, 17954. http://dx.doi.org/10.1103/PhysRevB.54.17954

24. Miyamoto, Y.; Nakada, K.; Fujita, M. Phys. Rev. B 1999, 59, 9858. http://dx.doi.org/10.1103/PhysRevB.59.9858

25. Jiao, L.; Zhang, L.; Wang, X.; Diankov, G.; Dai, H. Nature 2009, 458, 877. http://dx.doi.org/10.1038/nature07919 
26. Kosynkin, D. V.; Higginbotham, A. L.; Sinitskii, A.; Lomeda, J. R.; Dimiev, A.; Price, B. K.; Tour, J. M. Nature 2009, 458, 872.

http://dx.doi.org/10.1038/nature07872

27. Cano-Márquez, A. G.; Rodríguez-Macías, F. J.; Campos-Delgado, J.; Espinosa-González, C. G.; Tristán-López, F.; Ramírez-González, D.; Cullen, D. A.; Smith, D. J.; Terrones, M.; Vega-Cantú, Y. I. Nano Lett. 2009, 9, 1527. http://dx.doi.org/10.1021/n1803585s

28. Elías, A. L.; Botello-Méndez, A. s. R.; Meneses-Rodríguez, D.; Jehová González, V.; Ramírez-González, D.; Ci, L.; Muñoz-Sandoval, E.; Ajayan, P. M.; Terrones, H.; Terrones, M. Nano Lett. 2009, 10, 366.

29. Wang, X.; Dai, H. Nat. Chem. 2010, 2, 661. http://dx.doi.org/10.1038/nchem.719

30. Kato, T.; Hatakeyama, R. Nat. Nanotechnol. 2012, 7, 651. http://dx.doi.org/10.1038/nnano.2012.145

31. Hummers, W. S.; Offeman, R. E. J. Am. Chem. Soc. 1958, 80, 1339. http://dx.doi.org/10.1021/ja01539a017

32. Stankovich, S.; Dikin, D. A.; Dommett, G. H. B.; Kohlhaas, K. M.; Zimney, E. J.; Stach, E. A.; Piner, R. D.; Nguyen, S. T.; Ruoff, R. S. Nature 2006, 442, 282. http://dx.doi.org/10.1038/nature04969

33. Li, D.; Muller, M. B.; Gilje, S.; Kaner, R. B.; Wallace, G. G. Nat. Nanotechnol. 2008, 3, 101. http://dx.doi.org/10.1038/nnano.2007.451

34. Tung, V. C.; Allen, M. J.; Yang, Y.; Kaner, R. B. Nat. Nanotechnol. 2009, 4, 25. http://dx.doi.org/10.1038/nnano.2008.329

35. Tapaszto, L.; Dobrik, G.; Lambin, P.; Biro, L. P. Nat. Nanotechnol. 2008, 3, 397. http://dx.doi.org/10.1038/nnano.2008.149

36. Vo, T. H.; Shekhirev, M.; Kunkel, D. A.; Morton, M. D.; Berglund, E.; Kong, L.; Wilson, P. M.; Dowben, P. A.; Enders, A.; Sinitskii, A. Nat. Commun. 2014, 5, 3189. http://dx.doi.org/10.1038/ncomms4189

37. Vo, T. H.; Shekhirev, M.; Kunkel, D. A.; Orange, F.; Guinel, M. J. F.; Enders, A.; Sinitskii, A. Chem. Commun. 2014, 50, 4172. http://dx.doi.org/10.1039/c4cc00885e

38. Sakaguchi, H.; Kawagoe, Y.; Hirano, Y.; Iruka, T.; Yano, M.; Nakae, T. Adv. Mater. 2014, $26,4134$. http://dx.doi.org/10.1002/adma.201305034

39. Yang, X.; Dou, X.; Rouhanipour, A.; Zhi, L.; Räder, H. J.; Müllen, K. J. Am. Chem. Soc. 2008, 130, 4216. http://dx.doi.org/10.1021/ja710234t

40. Qian, H.; Negri, F.; Wang, C.; Wang, Z. J. Am. Chem. Soc. 2008, 130, 17970. http://dx.doi.org/10.1021/ja807803j 
41. Fogel, Y.; Zhi, L.; Rouhanipour, A.; Andrienko, D.; Räder, H. J.; Müllen, K. Macromolecules 2009, 42, 6878.

http://dx.doi.org/10.1021/ma901142g

42. Dössel, L.; Gherghel, L.; Feng, X.; Müllen, K. Angew. Chem., Int. Ed. 2011, 50, 2540. http://dx.doi.org/10.1002/anie.201006593

43. Schwab, M. G.; Narita, A.; Hernandez, Y.; Balandina, T.; Mali, K. S.; De Feyter, S.; Feng, X.; Müllen, K. J. Am. Chem. Soc. 2012, 134, 18169. http://dx.doi.org/10.1021/ja307697j

44. Kim, K. T.; Jung, J. W.; Jo, W. H. Carbon 2013, 63, 202. http://dx.doi.org/10.1016/j.carbon.2013.06.074

45. Konishi, A.; Hirao, Y.; Matsumoto, K.; Kurata, H.; Kishi, R.; Shigeta, Y.; Nakano, M.; Tokunaga, K.; Kamada, K.; Kubo, T. J. Am. Chem. Soc. 2013, 135, 1430. http://dx.doi.org/10.1021/ja309599m

46. Narita, A.; Feng, X.; Hernandez, Y.; Jensen, S. A.; Bonn, M.; Yang, H.; Verzhbitskiy, I. A.; Casiraghi, C.; Hansen, M. R.; Koch, A. H. R.; Fytas, G.; Ivasenko, O.; Li, B.; Mali, K. S.; Balandina, T.; Mahesh, S.; De Feyter, S.; Müllen, K. Nat. Chem. 2014, 6, 126. http://dx.doi.org/10.1038/nchem.1819

47. Chuvilin, A.; Bichoutskaia, E.; Gimenez-Lopez, M. C.; Chamberlain, T. W.; Rance, G. A.; Kuganathan, N.; Biskupek, J.; Kaiser, U.; Khlobystov, A. N. Nat. Mater. 2011, 10, 687. http://dx.doi.org/10.1038/nmat3082

48. Talyzin, A. V.; Anoshkin, I. V.; Krasheninnikov, A. V.; Nieminen, R. M.; Nasibulin, A. G.; Jiang, H.; Kauppinen, E. I. Nano Lett. 2011, 11, 4352. http://dx.doi.org/10.1021/n12024678

49. Cai, J.; Ruffieux, P.; Jaafar, R.; Bieri, M.; Braun, T.; Blankenburg, S.; Muoth, M.; Seitsonen, A. P.; Saleh, M.; Feng, X.; Müllen, K.; Fasel, R. Nature 2010, 466, 470. http://dx.doi.org/10.1038/nature09211

50. Bronner, C.; Stremlau, S.; Gille, M.; Brausse, F.; Haase, A.; Hecht, S.; Tegeder, P. Angew. Chem., Int. Ed. 2013, 52, 4422. http://dx.doi.org/10.1002/anie.201209735

51. Dötz, F.; Brand, J. D.; Ito, S.; Gherghel, L.; Müllen, K. J. Am. Chem. Soc. 2000, 122, 7707. http://dx.doi.org/10.1021/ja000832x

52. Britz, D. A.; Khlobystov, A. N.; Porfyrakis, K.; Ardavan, A.; Briggs, G. A. D. Chem. Commun. 2005, 37. http://dx.doi.org/10.1039/b414247k 